\title{
Stress induces the transcription of toxin-antitoxin systems but does not activate toxin
}

Michele LeRoux ${ }^{1}$, Peter H. Culviner ${ }^{1}$, Yue J. Liu ${ }^{1}$, Megan L. Littlehale ${ }^{1}$, Michael T. Laub ${ }^{1,2,3}$

${ }^{1}$ Department of Biology, Massachusetts Institute of Technology, Cambridge, MA 02139, USA

${ }^{2}$ Howard Hughes Medical Institute, Massachusetts Institute of Technology, Cambridge, MA 02139, USA

${ }^{3}$ Correspondence can be addressed to MTL: laub@mit.edu, 617-324-0418 


\begin{abstract}
Toxin-antitoxin (TA) systems are ubiquitous genetic elements in bacterial genomes, but their functions are controversial. Although they are frequently postulated to regulate cell growth following stress, few null phenotypes for TA systems have been reported. Here, we show that TA transcript levels can increase substantially in response to stress, but toxin is not liberated. We find that the growth of an Escherichia coli strain lacking 10 TA systems encoding endoribonuclease toxins is not affected following exposure to six stresses that each trigger TA transcription. Additionally, using RNA-sequencing, we find no evidence of mRNA cleavage following stress. Stress-induced transcription arises from antitoxin degradation and relief of transcriptional autoregulation. Importantly, although free antitoxin is readily degraded in vivo, antitoxin bound to toxin is protected from proteolysis, preventing release of active toxin. Thus, transcription is not a reliable marker of TA activity, and TA systems likely do not strongly promote survival following stress.
\end{abstract}




\section{Introduction}

Toxin-antitoxin (TA) systems are composed of a growth-inhibiting toxin and a cognate, neutralizing antitoxin. These systems are found in the majority of bacterial chromosomes and also on plasmids, phage, and other mobile elements, with many species encoding dozens of different systems. Despite their prevalence, the functions of most TA systems remain unknown. They have been suggested to promote stress tolerance, genome stability, persister cell formation, and programmed cell death, but the evidence supporting these functions is often limited, speculative, or controversial (Christensen et al., 2003; Gerdes and Maisonneuve, 2012; Harms et al., 2016; Hazan et al., 2004; Kolodkin-Gal and Engelberg-Kulka, 2006; Magnuson, 2007; Nigam et al., 2019; Ronneau and Helaine, 2019; Van Melderen and Wood, 2017; Wang et al., 2011; Yamaguchi et al., 2011).

TA systems are classified by the nature of the antitoxin, with the most well-characterized versions, termed type II systems, consisting of a protein that binds the cognate toxin to prevent its activity. These type II toxin-antitoxin pairs are encoded in the same operon and co-expressed (Fig. 1A). The targets of toxins from type II systems vary, but most inhibit a central cellular process such as translation or DNA replication (Yamaguchi et al., 2011). The direct targets have been identified by ectopically expressing individual toxins, which are often bacteriostatic and thus reversible when antitoxin levels are restored. Most antitoxins are small proteins that harbor unstructured or disordered regions and are thought to be substrates of proteases such as Lon and ClpP (Gerdes and Maisonneuve, 2012; Yamaguchi et al., 2011). Although toxin-antitoxin pairs are co-transcribed, antitoxins are usually translated at a higher rate, presumably to outpace antitoxin protein turnover and maintain sufficient levels for neutralizing toxin (Li et al., 2014). In addition to binding their cognate toxins, most antitoxins transcriptionally autoregulate by directly binding the TA operon promoter to repress transcription. Many TA systems exhibit so-called conditional cooperativity, in which low concentrations of toxin promote the binding of antitoxin to its promoter whereas higher concentrations of toxin disrupt binding (Overgaard et al., 2008).

The first type II TA system identified, CcdAB, is plasmid-encoded and was found to promote plasmid inheritance through post-segregational killing (Jaffé et al., 1985; Salmon et al., 1994). The antitoxin CcdA is both highly expressed and intrinsically unstable (Van Melderen et al., 1994). Thus, if the plasmid harboring $c c d A B$ is not segregated into a daughter cell, CcdA is degraded and 
cannot be replenished, leading to liberation of the $\mathrm{CcdB}$ toxin which can kill cells, thereby ensuring that plasmid-free cells do not survive.

Type II TA systems were subsequently found encoded on bacterial chromosomes. These systems do not stabilize chromosomes as with $\mathrm{CcdAB}$, raising the question of what function, if any, they provide cells. Chromosomal TA systems are typically assumed to have similar properties to CcdAB. However, antitoxin degradation rates have, to our knowledge, never been examined in their native contexts using pulse-chase experiments. Instead, antitoxin degradation is most commonly examined by high ectopic expression coupled with translation-shutoff experiments or by in vitro degradation assays (Cherny, 2005; Christensen et al., 2001; Prysak et al., 2009; Wang et al., 2011). Many studies have invoked TA systems as stress response elements, primarily because they are often transcriptionally upregulated in stressful conditions, including nutritional limitations, heat shock, and oxidative stress (Christensen-Dalsgaard et al., 2010; Muthuramalingam et al., 2016; Ronneau and Helaine, 2019). However, increased transcription of a TA system does not necessarily imply that toxin is liberated and active. Notably, there are very few reported cases of chromosomal TA systems that have null phenotypes in the stress conditions that lead to their increased transcription.

An early, influential study on the RelBE TA system found that transcription increased following nutritional stress and that global cellular translation rates were $\sim$ two-fold higher in a $\triangle$ relBE strain compared to the wild type in those same conditions, but the reproducibility of this small effect is unclear and no growth phenotype was reported for the $\triangle r e l B E$ strain (Christensen and Gerdes, 2004). A subsequent study examined a strain lacking five TA systems in E. coli, finding that it did not grow better following several different stresses (Tsilibaris et al., 2007). A study of the YefMYoeB system found that its toxin, YoeB, cleaves an ectopically expressed, artificial mRNA featuring multiple ribosome pause sites following heat shock, but did not see cleavage of select native transcripts or a YoeB-dependent growth phenotype (Janssen et al., 2015).

A potential breakthrough came when the Gerdes lab reported that the sequential deletion of 10 chromosomal TA systems in Escherichia coli $(\Delta 10 \mathrm{TA})$ significantly reduced the frequency of antibiotic-tolerant persister cells (Maisonneuve et al., 2011). Their subsequent work argued that guanosine penta- and tetra-phosphate, (p)ppGpp, the master regulator of the bacterial stringent response, induces TA systems by driving the accumulation of polyphosphate to stimulate the Lon 
protease to degrade antitoxins at an accelerated rate, thereby freeing toxins to promote persister cell formation (Maisonneuve et al., 2013). However, much of this work was retracted when it came to light that the $\Delta 10 \mathrm{TA}$ strain had acquired multiple $\phi 80$ prophage insertions that were responsible for the persister phenotypes observed (Goormaghtigh et al., 2018; Harms et al., 2017).

Since the discovery of chromosomal TA systems 30+ years ago, numerous studies have invoked a role in responding to stress, but there is little evidence that these systems alter cellular physiology during or after stress. To address this paradox, we set out to understand (i) how TA transcription increases following abiotic stress and (ii) whether these increases in transcription are associated with toxin activity. We determined that six diverse stresses induce TA transcription, often quite substantially. However, we find that a $\Delta 10 \mathrm{TA}$ strain (lacking $\phi 80$ prophages) does not grow any faster than a wild-type strain following these stresses as would be the case if stress activated any of these growth-inhibitory toxins. Additionally, using RNA-seq, we find no evidence of endoribonuclease toxin activity following stress. Through detailed study of two E. coli K12 TA systems, YefM-YoeB and MqsA-MqsR, we find that increases in TA transcription following stress arise from a relief of autoregulation. Stress triggers either a decrease in synthesis of toxin and antitoxin coupled to ongoing degradation of antitoxins, or it accelerates antitoxin degradation, though not in a ppGpp-dependent manner, as previously suggested (Christensen et al., 2001; Maisonneuve and Gerdes, 2014). In either case, antitoxin turnover relieves autoregulation, leading to transcriptional induction. Importantly, we demonstrate that free antitoxin is preferentially degraded relative to antitoxin in complex with toxin. Consequently, toxin remains inhibited and is not liberated. In sum, our results strongly suggest that although TA systems are transcriptionally induced by stress, they are likely not critical effectors of bacterial stress responses, as is frequently asserted. 


\section{Results}

\section{Type II TA systems are transcriptionally induced by diverse stress conditions}

Numerous studies have reported increased transcription of type II TA systems in response to diverse stress conditions. To determine the kinetics of these transcriptional responses, we subjected E. coli to two well-studied stresses, the stringent response to amino acid starvation, which can be rapidly induced via addition of serine hydroxamate (SHX), and translation inhibition, induced by treatment with chloramphenicol. Consistent with previous reports (Christensen-Dalsgaard et al., 2010; Muthuramalingam et al., 2016; Ronneau and Helaine, 2019; Shan et al., 2017), we found an increase of at least 6-fold in the mRNA levels of three type II TA systems in E. coli MG1655, $m q s R A$, relBE, and $y e f M-y o e B$, at both 15 and 30 minutes after treatment using quantitative reverse transcription PCR (qRT-PCR) (Fig. 1B-C).

Based on these data, we selected the 30-minute time point for subsequent qRT-PCR experiments, and examined the expression of 10 type II systems of E. coli MG1655 in response to a larger panel of stresses including amino-acid starvation (SHX), translation inhibition (chloramphenicol), DNA synthesis inhibition (trimethoprim), oxidative stress (hydrogen peroxide), cell-wall synthesis inhibition (carbenicillin), acid shock $(\mathrm{pH} 4)$, heat shock (shift from $30^{\circ} \mathrm{C}$ to $45^{\circ} \mathrm{C}$ ), and proteotoxic stress $(\Delta d n a K)($ Fig. 1D). We saw significant increases in antitoxin transcription, in some cases exceeding 50-fold. Although the magnitude of the responses varied across TA systems and not all systems responded to all stresses, each stress except carbenicillin elicited a transcriptional response in the majority of E. coli TA systems (Fig. 1D, Fig. S1).

\section{Type II TA systems do not affect growth following diverse stress conditions}

An increase in TA transcription during stress has frequently been interpreted to mean that TA systems are 'active' under these conditions. We reasoned that if toxin were released following a stress, we should see a TA-dependent decrease in growth during or immediately after the stress. To test this hypothesis, we used a recently constructed $\Delta 10 \mathrm{TA}$ strain in which each of 10 type II TA systems in E. coli MG1655 have been deleted to examine growth following a stress (Goeders et al., 2013; Goormaghtigh et al., 2018). This strain was verified as lacking the prophage contaminant found in a different, prior version of this strain. For each stress that induced TA transcription in Figure 1, we grew the wild-type and $\Delta 10 \mathrm{TA}$ strains to early exponential phase and 
then split each culture, treating half with a given stress for one hour while leaving the other half untreated for one hour (Fig. 2A). The cells were then washed to remove the stress agent and diluted into fresh medium. The growth of each culture was then measured for 8-10 hours. Despite the fact that each of these stresses induces TA transcription (Fig. 1D), none of them led to a difference in growth rate between the wild-type and $\Delta 10 \mathrm{TA}$ strain for up to 8 hours (Fig. 2B-I). The lag phase of the $\Delta 10 \mathrm{TA}$ strain was increased following hydrogen peroxide treatment compared to the wildtype (Fig. 2E); however, this is opposite of what is expected if toxins were liberated, and it may result from another mutation acquired in construction of the $\Delta 10 \mathrm{TA}$ strain. To rule out the possibility that small changes in growth were undetectable in these experiments, or that a single stress treatment was insufficient to stimulate detectable toxin activity, we also performed a competition experiment in which differentially labeled wild-type and $\Delta 10 \mathrm{TA}$ strains were mixed at a 1:1 ratio and subjected to two cycles of chloramphenicol treatment. The $\Delta 10 \mathrm{TA}$ strain displayed no competitive advantage or disadvantage, again indicating that toxins are not liberated by this stress (Fig. 2J).

To test for differences in growth during the stress itself, we also monitored growth at $44{ }^{\circ} \mathrm{C}$ (Fig. $2 \mathrm{~K})$, and immediately after adding SHX and chloramphenicol without washing out the stress agent (Fig. 2L-M). In none of these conditions did we observe significant differences in growth between the wild-type and $\Delta 10 \mathrm{TA}$ strains. We also examined growth after longer exposure (4 hours) to a growth-inhibitory concentration of chloramphenicol $(75 \mu \mathrm{g} / \mathrm{mL})$ followed by washout of the stressor, and saw a very subtle growth difference (Fig. $2 \mathrm{~N}$ ). Although a minor difference was evident after these extended periods of chloramphenicol stress, TA transcription increased within minutes in the same conditions (Fig. 1B-C). Thus, we conclude that toxins are not strongly or significantly activated by stress and that transcriptional activation is not a reliable proxy for toxin activity.

\section{RNA sequencing reveals no evidence of toxin activity}

For a more direct assay of toxin activity, we turned to RNA sequencing (RNA-seq) as each of the 10 toxins deleted in the $\Delta 10 \mathrm{TA}$ strain are endoribonucleases. The endoribonuclease activity of a toxin can be quantitatively measured by comparing RNA-seq fragment-densities between cells expressing a given toxin and cells not expressing the toxin (Culviner and Laub, 2018) (Fig. 3A). A ratio of fragment density $+/$ - a given toxin can be calculated for each nucleotide in each 
transcript. Regions where the ratio is negative may indicate toxin cleavage, or could reflect changes in transcript levels or stability that arise after toxin expression. To distinguish between toxin activity and other changes to mRNA abundance, we focused on areas with steep "valleys", which are characteristic of toxin cleavage events (Culviner and Laub, 2018). Because the toxin MazF one of the toxins deleted in the $\Delta 10$ strain - has been extensively characterized and has a welldefined cleavage motif, we focused the first part of our analysis on this toxin. An example profile of fragment density following the induction of MazF for $10 \mathrm{~min}$ is shown in Fig. 3A (Culviner and Laub, 2018). This profile exhibits a clear signature of MazF cleavage: a region with a low ratio, $\sim 30$ nucleotides into the $r p l S$ transcript, followed by a steep increase that coincides with a highscoring MazF motif (Fig. 3A, bottom). This high positive slope in the ratio profile occurs because MazF typically generates one product that can be rapidly degraded by 3 '-5' exonucleases and one product that is more stable as E. coli lacks a 5'-3' exonuclease.

We recently generated RNA-seq data for cells expressing each of 9 endoribonuclease toxins in $E$. coli for $5 \mathrm{~min}$, including MazF (see Methods). These toxins are those deleted in the $\Delta 10 \mathrm{TA}$ strain; YafQ was excluded as no cleavage or growth defect was detected following its overexpression. To test whether stress induces activity of any the endoribonuclease toxins, we performed RNA-seq on wild-type and $\Delta 10 \mathrm{TA}$ cells following 15, 30, and $60 \mathrm{~min}$ of exposure to a concentration of chloramphenicol that results in high TA transcription (Fig. 1). We then calculated a ratio of fragment density at each nucleotide in each transcript for wild-type v. $\Delta 10 \mathrm{TA}$ cells. These ratios were compared to the ratios generated following the overexpression of each toxin. We examined regions with strong signatures of cleavage following toxin overexpression, but found no evidence of TA-dependent mRNA cleavage following chloramphenicol treatment at any of the time points tested (Fig. 3A-C, Fig. S2A-I). The ratios of fragment density for wild type v. $\Delta 10 \mathrm{TA}$ following stress did not exhibit large valleys like those seen after ectopically producing an individual toxin.

We also considered the possibility that stress leads only to very modest cleavage or cleavage in only a subset of cells. If so, we would expect ratios of fragment density for wild type v. $\Delta 10 \mathrm{TA}$ to have a similar overall shape as those produced following ectopic toxin expression, but with shallower valleys. For instance, in cells in which MazF expression is titrated to levels that allow continued cell growth (Culviner and Laub, 2018), the $+/$ - toxin profiles retain very similar overall shapes, but with a reduction in magnitude (Fig. S3A). A close inspection of the cleavage profiles 
following chloramphenicol treatment did not reveal any substantial similarity to the profiles generated after ectopic toxin expression (Fig S3A).

To more systematically assess whether chloramphenicol stress activates the endoribonuclease toxins, we first selected the transcripts containing the largest (top 5\%) slopes in the ratio profiles resulting from expression of MazF, thereby defining a set of 'MazF targets'. We then (i) identified the maximum slope for each of these transcripts in the profiles generated by comparing wild type v. $\Delta 10 \mathrm{TA}$ in chloramphenicol and (ii) calculated the mean of these slopes (see red dots in Fig. 3D). We repeated this same process but for 10,000 different, random sets of transcripts to produce a distribution reflecting the expected value of mean slope maxima for a random set of transcripts. We could then calculate a z-score for the mean slope maxima of the MazF targets (Fig. 3D). A large z-score would indicate that MazF targets are cleaved more following chloramphenicol stress than expected by chance. However, the z-scores for MazF targets in the three chloramphenicol data sets were $-2.0,0$, and 0.9 for the 15, 30, and 60 minute time-points respectively (Fig. 3D) indicating no significant evidence of cleavage. As a control, we confirmed that the MazF targets do yield a high z-score of 16.1 when compared to a replicate of the MazF expression data rather than chloramphenicol stress (Fig. 3D).

We performed this same overall procedure for the targets of the other 8 endoribonuclease toxins. We did not observe high z-scores for any of the other toxin targets in any of the three chloramphenicol data sets (Fig. 3E). YhaV had the highest z-scores of 1.8, 2.3, and 1.5, but inspection of the profiles for the transcripts defined as YhaV targets did not reveal signatures of cleavage in wild type v. $\Delta 10 \mathrm{TA}$ in chloramphenicol, i.e. the shape of these profiles did not resemble those resulting from YhaV induction (Fig. S3B). Taken together, these results support our conclusion that there is no substantial activation of toxins in cells exposed to chloramphenicol for up to 60 minutes.

The analyses above are based on the assumption that large slopes in the profiles generated by expression individual toxins appropriately capture cleavage events. To consider the possibility of cleavage events that do not result in steep slopes (e.g. if the $5^{\prime}$ end created by cleavage is not stable, and leads to a wide valley in the $+/$ - toxin ratio plots), we repeated our analysis using minima within transcripts rather than slopes to define toxin targets; in this analysis, low z-scores would indicate cleavage of toxin targets in the chloramphenicol data sets (Fig. S3D-E). However, the 
lowest z-score in the chloramphenicol treatment data set was only -1.9, for YafO at 60 min (Fig. $\mathrm{S} 3 \mathrm{E})$.

Taken all together, our results indicate no clear, detectable evidence of cleavage by any endoribonuclease toxin in the wild type relative to the $\Delta 10$ strain following 15,30 , or 60 minutes of chloramphenicol stress. These findings support our growth measurements also indicating that toxins are not active following stress, despite their strong transcriptional induction.

\section{Transcriptional induction results from a relief of autoregulation}

We wanted to understand, at a mechanistic level, how TA systems are transcriptionally induced without apparently liberating toxin. We focused on three TA systems: YefM-YoeB, MqsRA, and RelBE. To test whether the stress-induced transcription of these systems stems from a loss of transcriptional autoregulation, we first generated chromosomally-encoded antitoxin alleles with mutations in residues that are required for DNA-binding but that do not affect the ability of the antitoxin to neutralize its cognate toxin: $m q s A(N 97 A / R 101 A), \operatorname{relB}(R 7 A)$, and yefM(R10A) (Bailey and Hayes, 2009; Brown et al., 2011; Overgaard et al., 2009) hereafter referred to as $m q s A^{*}$, relB*, and $y$ ef $M^{*}$, respectively. As expected, baseline expression of these transcripts was elevated compared to the wild-type alleles (Fig. 4A). We then tested whether these strains still mounted a transcriptional response to chloramphenicol stress. In each case, the DNA-binding mutant no longer exhibited a large increase in transcription as seen with the wild type (Fig. 4B). These results indicate that the transcriptional responses seen in wild-type cells reflect a relief of autoregulation, likely resulting from an increase in antitoxin degradation.

To investigate whether antitoxin degradation was required for the stress-dependent increases in TA transcription, we screened a panel of E. coli protease deletion strains for changes in antitoxin transcript levels following chloramphenicol treatment. Although Lon is often involved in degrading antitoxins, other proteases have been implicated (Aizenman et al., 1996; Christensen et al., 2001; Janssen et al., 2015; Wang et al., 2011). Because an ftsH deletion results in a lethal dysregulation of lipopolysaccharide in $E$. coli it can only be generated in a strain carrying a suppressor mutation, $s f h C$; we thus compared the W3110 sfhC $\Delta f t s H$ strain to the W3110 sfhC background (Fig. 4C, bottom two rows) (Ogura et al., 1999). Strikingly, the induction of antitoxin transcription by chloramphenicol was largely abolished in a lon deletion strain for each of the three TA systems examined (Fig. 4C, Fig. S4). A small reduction in transcription was also evident in 
the $\operatorname{clpXP}$ and ftsH deletion strains, indicating that these proteases may contribute to the degradation of these antitoxins (Fig. 4C, Fig. S4). Collectively, our results indicate that the increase in TA transcription following stress arises from antitoxin degradation, primarily by Lon, and a consequent relief of autoregulation.

\section{Transcriptional induction of TA systems does not require the stringent response or ppGpp}

A previous model posited that Lon is stimulated to degrade antitoxins at a higher rate following the accumulation of ppGpp (Fig. 5A) (Maisonneuve and Gerdes, 2014). This model claimed that ppGpp promoted the accumulation of polyphosphate, which then directly stimulated Lon to degrade antitoxins, thereby liberating toxins to promote persistence. While results related to persistence were cited in a retraction of this paper, the data related to Lon activation were not and recent literature continues to invoke a connection between ppGpp and TA systems (ChristensenDalsgaard et al., 2010; Muthuramalingam et al., 2016; Ronneau and Helaine, 2019). Thus, we wanted to test whether the stringent response and ppGpp are required for the transcriptional activation of TA systems.

We showed in Figure 1 that treating cells with SHX, which stimulates ppGpp synthesis, produces a strong transcriptional response. However, treating cells with the antibiotic chloramphenicol, which leads to an accumulation of charged tRNAs that should inhibit RelA to reduce cellular ppGpp levels (Kaplan et al., 1973; J. Sokawa and Y. Sokawa, 1978), also stimulates TA transcription. To determine the levels of ppGpp in our experimental conditions, we treated cells with either SHX or chloramphenicol as before (Fig. 1), and then directly measured ppGpp levels using anion exchange chromatography. After 15 min of SHX treatment, ppGpp levels had reached approximately $2.5 \mathrm{nmol} / \mathrm{OD}_{600}$, approximately 27 -fold higher than in untreated cells, whereas chloramphenicol treatment reduced ppGpp levels to less than $0.01 \mathrm{nmol} / \mathrm{OD}_{600}$, approaching the limit of detection (Fig. 5B-C). We then asked whether cells that had lost the ability to produce polyphosphate - which was proposed to directly activate Lon (Fig. 5A) - could still respond transcriptionally to stress. To test this idea, we used a strain harboring deletions of the genes polyphosphate kinase ( $p p k)$ and polyphosphate phosphatase ( $p p x)$, which encode the enzymes responsible for producing and degrading polyphosphate. We found that when this strain was treated with either SHX or chloramphenicol, TA transcription was still induced (Fig. 5D-E). The magnitudes of induction were slightly less than in a wild-type strain, possibly because $\Delta p p k \Delta p p x$ 
cells respond differently to high levels of ppGpp. Nevertheless, this strain exhibited substantial increases in TA transcription following translational stress, regardless of whether ppGpp is produced. Thus, our results indicate that neither ppGpp nor polyphosphate is required for the transcriptional induction of TA systems.

\section{Translation inhibition can accelerate antitoxin degradation}

To more directly test whether ppGpp affects the stability of antitoxins, as postulated previously (Fig. 5A), we used pulse-chase assays to measure the degradation rates of the antitoxins YefM and MqsA in cells experiencing high or low ppGpp levels, induced by treatment with SHX and chloramphenicol, respectively. Native antitoxin levels were too low to be detected by pulse-chase, so we individually cloned the yefM-yoeB and $m q s R A$ operons - including the native promoter in each case - onto a medium-copy plasmid. There was no substantial increase in the rate of degradation for YefM or MqsA in SHX compared to chloramphenicol (Fig. 5F-G). In fact, MqsA was degraded slightly faster in the low ppGpp (chloramphenicol) condition than in the high ppGpp (SHX) condition (Fig. 5G). These results directly contradict those reported previously (Maisonneuve and Gerdes, 2014), and further indicate that the degradation of antitoxins following stress does not depend on ppGpp.

Our results indicate that TA transcription following chloramphenicol or SHX treatment likely arises from (i) an inhibition of protein synthesis by these stressors and (ii) antitoxin degradation by Lon, leading to a relief of TA autoregulation. In some cases, these stresses accelerate degradation beyond basal, pre-stress rates, though in a ppGpp-independent manner (Fig. 5F-G). Notably, many prior studies of antitoxin stability have relied on Western blotting of chloramphenicol-treated cells, so-called translational shut-off assays. Our finding that chloramphenicol-treatment significantly accelerates the degradation of YefM (Fig. 5F) and moderately increases the degradation of MqsA (Fig. 5G) indicate that such shutoff assays may confound the accurate assessment of degradation rates.

\section{Heat shock induces transcription of TA systems by increasing antitoxin degradation}

In contrast to chloramphenicol and SHX treatment, some stresses that lead to TA transcription, e.g. heat shock (Fig. 1), do not inhibit protein synthesis. In these cases, the stress presumably must accelerate the degradation of antitoxins to produce the transcriptional induction observed. To test this hypothesis, we used pulse-chase analyses to examine the effect of heat shock on antitoxin 
stability following a shift from 30 to $45^{\circ} \mathrm{C}$. Indeed, we found that heat shock significantly increased the rate of YefM degradation (Fig. 6A, S5A), consistent with prior studies (Janssen et al., 2015). Because heat shock does not inhibit translation, the increased degradation of YefM and consequent relief of autorepression increases antitoxin transcription (Fig. 1) and should also lead to an increase in antitoxin protein levels. To test this prediction, we examined YefM levels following heat shock. We were unable to detect YefM expressed from its native promoter by Western blot, so we radiolabeled proteins to steady state and measured YefM levels by immunoprecipitation and phosphorimaging. Following heat shock, the levels of YefM increased approximately 2-fold over 60 minutes, as predicted (Fig. 6B, S5B).

Notably, wild-type cells sustain transcriptional induction (Fig. 1) and continue to accumulate antitoxin after stress (Fig. 6B), indicating that the newly synthesized antitoxin does not immediately feedback and inhibit transcription. This sustained induction likely results from the conditional cooperativity property of many TA systems including YefM-YoeB (Fig. 6C). In vitro studies of YefM-YoeB found that when toxin:antitoxin ratios are $\sim 1: 2$, the toxin promotes binding of antitoxin to its own promoter; however, at a toxin:antitoxin ratio $>2: 1$, the toxin disrupts DNA binding by the antitoxin (Kedzierska et al., 2006). Thus, we hypothesized that an increase in the toxin:antitoxin ratio occurs after heat shock, producing the sustained transcriptional induction observed (Fig. 6C). Our attempts to directly measure the toxin:antitoxin ratio were unsuccessful as adding an epitope tag to the toxin affected the toxin:antitoxin interaction. We therefore took a different approach to testing our hypothesis. We reasoned that an increase in the toxin:antitoxin ratio would occur only if free antitoxin were preferentially degraded compared to antitoxin in complex with toxin. Indeed, we noticed that although SHX, chloramphenicol, and heat shock all increased the degradation rate of YefM, $\sim 20 \%$ of the initially labeled pool remained stable for up to 60 minutes (Fig. 5F, Fig. 6A). We suspected that this stable pool is YefM antitoxin bound to YoeB toxin.

To test whether YoeB affects YefM stability, we used pulse-chase analysis to measure the stability of YefM expressed alone from a plasmid, or co-expressed with its cognate toxin YoeB, in the absence of any stress. We found that YefM was significantly less stable in the absence of YoeB, with labeled YefM nearly undetectable after 15 minutes (Fig. 6D, Fig. S5C). The preferential degradation of free antitoxin supports a model in which the ratio of toxin to antitoxin increases following a stress that promotes antitoxin degradation. Additionally, the relative stability of 
toxin:antitoxin complexes ensures that little to no free toxin is released. Taken together, these results indicate that although stress can trigger a significant and sustained transcriptional induction of yefM-yoeB, it does not result in the liberation of significant levels of free toxin (Fig. 6C).

Finally, because the increase in YefM levels following heat shock results from a relief of autoregulation, it should depend on the DNA-binding ability of YefM. To test this idea, we examined strains expressing the DNA-binding deficient YefM variant, YefM*. We first measured changes in YefM* degradation rates in response to heat shock, and found that, as with the wildtype protein, heat shock accelerated the degradation of YefM* compared to untreated cells (Fig. $6 \mathrm{E}$, Fig. S5D). We then measured overall protein levels of YefM* by immunoprecipitation and found that the levels did not increase as with wild-type YefM, but instead decreased to about 80\% that seen in untreated cells (Fig. 6F, Fig. S5E). This result confirms that heat shock in wild-type cells triggers accelerated degradation of YefM antitoxin, leading to a relief of transcriptional autoregulation.

We also tested how heat shock affects the MqsA-MqsR TA system. As with YefM, we observed an increase in MqsA antitoxin degradation following heat shock (Fig. 6G, Fig. S5F). When we examined MqsA levels by Western blot, we detected a six-fold increase in MqsA under heat shock conditions compared with untreated cells (Fig. 6H, Fig. S5G). The MqsA-MqsR system does not exhibit conditional cooperativity. However, like the YefM-YoeB system, as the concentration of MqsR toxin increases, it disrupts the binding of MqsA to DNA (Brown et al., 2013). Thus, the sustained induction of $m q s R A$ following stress likely arises, as with YefM-YoeB, from increased degradation of MqsA leading to increases in the ratio of MqsR:MqsA, with MqsA bound to MqsR unable to efficiently autoregulate transcription (Fig. S5H). We noted that MqsA degradation also plateaued like YefM following heat shock, leveling off at approximately $60 \%$ initial levels, suggesting that a pool of MqsA bound to MqsR may be recalcitrant to degradation. Consistent with this idea, MqsA is degraded more rapidly when expressed alone rather than with its cognate toxin, MqsR (Fig. 6I, Fig. S5I). As with YefM, the preferential degradation of free MqsA antitoxin relative to MqsA bound to MqsR likely helps ensure that no toxin is liberated following stress (Fig $\mathrm{S} 5 \mathrm{H})$.

We also measured the degradation and cellular abundance of MqsA* following heat shock. As with YefM*, MqsA* was degraded more rapidly after heat shock, and cellular levels decreased 
approximately 2-fold over the course of 60 minutes, indicating that cellular increases in MqsA abundance result from a relief of autoregulation (Fig. 6K, Fig. S5K). We also found that MqsA* is significantly more labile than wild-type MqsA both at $30{ }^{\circ} \mathrm{C}$ and following heat shock, suggesting that DNA binding may normally help protect MqsA from degradation, as reported for other Lon substrates (Pruteanu et al., 2007; Shah and Wolf, 2006).

\section{Chromosomal TA systems differ from plasmid-based systems}

Our results suggest that the E. coli chromosomal TA systems are fundamentally different from plasmid-based systems. For plasmid-based systems such as $\mathrm{CcdAB}$, toxins are presumably liberated when synthesis abruptly stops following plasmid loss (Van Melderen et al., 1994) as plasmid-free cells are inviable. Our results suggest that a similar mechanism would not be sufficient to activate chromosomal TA systems, as these antitoxin-toxin complexes are relatively resistant to proteolysis, preventing toxin from being liberated. To directly compare the chromosomal systems to $\mathrm{CcdAB}$ in a scenario similar to plasmid loss, we generated strains in which the only copy of MqsRA, YefM-YoeB, or CcdAB was under control of the arabinose promoter, $\mathrm{P}_{\text {ara }}$, on the chromosome. Each strain was either grown in the presence of arabinose to express the TA system or not induced. We then rapidly shut off new synthesis (analogous to plasmid loss) by washing cells and releasing them into medium containing glucose, which represses $\mathrm{P}_{\text {ara }}$ (Fig. 7A). As expected, cells harboring $c c d A B$ showed a clear decrease in growth rate $\sim 4$ hrs after inhibiting synthesis compared to cells in which CcdAB was not initially induced (Fig. 7B). The slow time scale of CcdAB activation is consistent with early studies of plasmid loss (Jaffé et al., 1985), and likely represents a combination of time required for antitoxin turnover and the time needed for cells to adapt to a change in carbon source (from glycerol + arabinose to glucose). In contrast to $\mathrm{CcdAB}$, we saw no changes in growth following the induction and subsequent repression of either MqsRA or YefM-YoeB (Fig. 7C-D). These findings are consistent with our pulse-chase studies (Fig. 5-6) indicating that the antitoxins MqsR and YefM are, in contrast to CcdA, maintained in relatively stable complexes with their toxins such that blocking new synthesis does not lead to their rapid decay and a consequent release of growth-inhibiting toxin. 


\section{Discussion}

Contrary to the widely-held view that TA systems function as stress-response modules, we found that abiotic stress can trigger transcription of chromosomally encoded TA systems in E. coli, but that significant, growth-inhibitory levels of free, active toxin are not generated by these stresses. Numerous prior studies have interpreted the robust increases in TA transcription to mean that (i) antitoxin levels were depleted and (ii) that this results in release of active toxin (ChristensenDalsgaard et al., 2010; Muthuramalingam et al., 2016; Ronneau and Helaine, 2019). Our results support the initial inference that stress can accelerate antitoxin turnover and thereby induce transcription by relieving autorepression. However, we find that, despite increases in antitoxin degradation rates, antitoxin pools are never fully depleted, and thus toxin is never actually freed. Using two different measures of toxin activity: changes in growth, and mRNA cleavage, we find no evidence that the 10 endoribonuclease toxins of $E$. coli are significantly activated by numerous, diverse abiotic stresses on time-scales coincident with major changes in their transcription.

How can an increase in antitoxin turnover occur, yet not liberate toxin? Two major factors contribute: (i) antitoxins are typically much more abundant than their cognate toxins and (ii) free antitoxin is preferentially degraded relative to antitoxin bound to its cognate toxin. Thus, in cases where a stress, e.g. heat shock, triggers accelerated degradation of antitoxin, the ratio of toxin:antitoxin increases. For both YefM-YoeB and MqsA-MqsR, increases in this ratio are known to disrupt autorepression by the antitoxin (Brown et al., 2013; Kedzierska et al., 2006), thereby explaining the sustained transcriptional induction. Importantly, because antitoxin bound to toxin is recalcitrant to degradation, there is little to no free toxin released, despite the transcriptional induction. In cases where a stress does not accelerate degradation but also blocks new synthesis, e.g. chloramphenicol treatment, the same logic applies. Free antitoxin is preferentially degraded, leading to an increase in the ratio of toxin:antitoxin, which will drive transcriptional induction, but without commensurate increases in protein levels given the block to synthesis. In sum, our results now provide a mechanistic basis for understanding how diverse stresses can trigger transcriptional induction, and in some cases increased protein levels, without liberating active toxin.

\section{Stress-induced changes in antitoxin degradation}

How is Lon-dependent degradation of antitoxins stimulated by diverse stresses? Early studies of Lon suggested that it can be activated by polyphosphate to degrade ribosomal proteins (Kuroda et 
al., 2001). A subsequent study extended this idea, postulating that the ppGpp made during amino acid starvation can stimulate polyphosphate to activate Lon-dependent degradation of antitoxins (Maisonneuve et al., 2013). However, we found no evidence that either ppGpp or polyphosphate are required for the increased degradation of antitoxins in stressful growth conditions. An alternative possibility is that because Lon normally degrades misfolded proteins that arise during translation (Mahmoud and Chien, 2018; Van Melderen and Aertsen, 2009), whenever the abundance of misfolded or incompletely synthesized peptides is substantially reduced, Lon may be more accessible to lower affinity substrates, such as antitoxins. The relatively long half-life of many antitoxins (>20 min) compared to some other Lon substrates (e.g. SulA, $\sim 1 \mathrm{~min}$ ) (Mizusawa and Gottesman, 1983), suggests that they may indeed be lower affinity Lon substrates, and it is known that Lon saturation affects the rate at which it degrades lower affinity substrates (Dervyn et al., 1990). Most of the stresses we tested were severe enough that protein translation was likely either fully or strongly inhibited. Exceptions to this include heat shock and the deletion of dnaK, although both of these conditions, which lead to proteotoxic stress, are known to stimulate Lon (Janssen et al., 2015; Jonas et al., 2013). A second possibility is that a Lon activator could become more abundant during stress. A subset of Lon substrates can function as activators of Lon in Yersinia pestis and Caulobacter crescentus (Jonas et al., 2013; Puri and Karzai, 2017). Because we saw an increase in antitoxin degradation even when translation is fully inhibited, this is a less likely scenario. However, we cannot rule out that a Lon activator is stabilized or becomes more abundant by a synthesis-independent mechanism.

Our studies of antitoxin degradation have also uncovered two important technical considerations when measuring protein half-life. First, the fact that antitoxins can be degraded faster, as measured by pulse-chase analysis, when cells are exposed to translation-inhibiting antibiotics is of broad relevance, given the common practice of measuring protein turnover by Western blotting after treating cells with antibiotics such as chloramphenicol to shutoff new translation. Because Lon activity increases in response to such antibiotics, this method may significantly overestimate the lability of some Lon substrates. A second important finding is that the half-life of an antitoxin depends on how it is produced. For instance, we found that the half-life of an antitoxin can depend strongly on whether its cognate toxin is being co-expressed or not (Fig. 6J). Similarly, we found that interactions with DNA can significantly affect the half-life of antitoxins, with point mutations that disrupt DNA-binding by antitoxins leading to increased lability (Fig. 6G and 6J). Similar 
effects have been documented for other Lon substrates (Ahn and Baker, 2016; Pruteanu et al., 2007; Shah and Wolf, 2006).

\section{Functions of toxin-antitoxin systems}

Our results demonstrate that diverse stresses can trigger increased degradation of antitoxins and that this relieves autoregulation to drive transcriptional induction of TA operons. However, these dynamics do not lead to the liberation of significant amounts of active toxin. If free toxin were being produced at appreciable levels, we would have seen a TA-dependent suppression of, or delay in, growth following the application of a stress. However, we observed no detectable reduction in the growth of wild-type cells compared to cells lacking 10 TA systems following heat shock, acid shock, or treatment with chloramphenicol, hydrogen peroxide, trimethoprim, or SHX (Fig. 2). As the 10 TA systems deleted each feature an endoribonuclease toxin, we also used RNA sequencing to test whether there is any evidence of toxin activity, but no cleavage of mRNA was detected (Fig. 3). It could be that TA systems are activated only in a very tiny fraction of cells experiencing a given stress such that no changes in bulk growth or RNA cleavage can be detected. But such an argument would still mean that TA systems are not being used as general stress-response modules.

How, then, do toxins ever get activated? Presumably, antitoxins must be dissociated (with or without degradation) from their cognate toxins, but the conditions that trigger such dissociation and, consequently, activation of a toxin remain unclear for most chromosomally-encoded TA systems. We find that the chromosomal type II TA systems function differently from the wellcharacterized plasmid-borne TA system CcdAB that inhibits cell growth upon plasmid loss (Van Melderen et al., 1994). The cessation of $c c d A B$ expression following plasmid loss, coupled with the relative instability of $\mathrm{CcdA}$, leads to the liberation of $\mathrm{CcdB}$ toxin, an effect that can be recapitulated by shutting off synthesis of $c c d A B$ (Fig. 7B). In contrast, we found that inhibiting synthesis of the chromosomally-encoded $m q s R A$ and yefM-yoeB systems was not sufficient to activate toxin, as measured by cell growth.

It remains to be seen whether plasmid-borne systems have different roles from chromosomal systems, or whether chromosomal systems are plasmid-derived systems that have lost, or are in the process of losing, their function over evolutionary time. We think the latter is unlikely because all the E. coli toxins have retained full activity. Another possibility is that the chromosomal systems in E. coli are activated by the same mechanism as CcdAB, but that they have slower off 
rates, and therefore require considerably longer timescales for toxin to be freed. One result in line with this model is the observation that the DinJ-YafQ system may protect cells against cefazolin or tobramycin when they are grown as biofilms, but not when grown in liquid (Harrison et al., 2009). Biofilms take several days to develop, and cells at the interior of the biofilm experience nutrient-poor conditions that may be sufficiently stressful to stimulate antitoxin degradation and prevent synthesis of new antitoxin over extended periods of time.

It is also conceivable that additional signals are needed to destabilize chromosomally-encoded TA complexes. Such a signal might arise under conditions not tested here, e.g. a biotic stress. A biotic stress could consist of phage infection, bacterial residence within a eukaryotic cell, or interbacterial antagonism within a microbial community. Each of these environments or conditions could lead to the co-occurrence of multiple stresses that induce toxin activity or the production of an unknown signal that alone is sufficient to induce toxins. Notably, the most recently discovered type II TA system in K12 E. coli, RnlAB, plays a clear role in defending E. coli against T4 phage infection, indicating that this system must somehow become active during phage infection (Koga et al., 2011), but the mechanism responsible is not known. Additionally, the chromosomal type II TA systems in Salmonella enterica ser. Typhimurium help promote survival within macrophages, indicating that they are active in this environment (Helaine et al., 2014), but again a mechanism of induction is not known.

In sum, our results emphasize that TA systems are unlikely to be critical or conserved components of bacterial stress response systems. Additionally, our findings strongly underscore the notion that the transcriptional activation of TA systems is not a reliable marker for activity. Specific assays of toxin activity such as the RNA-seq method used here to probe endoribonuclease activity will be crucial for identifying and characterizing the bona fide inducers of TA systems. 


\section{Acknowledgements}

We thank M. Guzzo, K. Gozzi, S. Jones, and M. Guo for comments on the manuscript and C. Aakre and D. Huang for helpful discussions. M. LeRoux was a Simons Foundation Fellow of the Life Sciences Research Foundation. This work was funded by an NIH grant to M.T.L. (R01GM082899), who is also an Investigator of the Howard Hughes Medical Institute.

\section{Author Contributions}

M.L performed all experiments. P.H.C. helped with RNA-seq experiments and analysis. Y.J.L. helped with qRT-PCR experiments and M. Littlehale helped with growth studies. M.L. and M.T.L designed experiments, analyzed data, prepared figures, and wrote the manuscript.

\section{Declaration of Interests}

The authors declare no competing interests. 


\section{Figure Legends}

\section{Figure 1. TA systems are transcriptionally induced by diverse stresses.}

(A) Schematic summarizing key properties of toxin-antitoxin systems. Toxin and antitoxin are cotranscribed from a single operon, but the antitoxin is translated at a higher rate. Antitoxins bind to and repress their own promoters. Antitoxins are also substrates of cellular proteases such as Lon. (B-C) Changes in antitoxin transcript level in response to serine hydroxamate (SHX) (B) or chloramphenicol (chlor) (C) as measured by qRT-PCR at the indicated time points. Antitoxin levels were normalized to the housekeeping gene gyrA and each stress normalized to measurements made for untreated cells.

(D) Difference in antitoxin transcript levels between cells exposed to stress for 30 minutes compared to untreated cells as measured by qRT-PCR.

Data are the average of 3 biological replicates with error bars indicating the S.D.

Also see Figure S1.

Figure 2. TA systems do not inhibit growth following diverse stress conditions.

(A) Experimental design for panels A-J to measure the growth of wild-type and $\Delta 10 \mathrm{TA}$ strains following stress exposure.

(B-C) Growth curves for WT and $\triangle 10 \mathrm{TA}$ strains grown at $30^{\circ} \mathrm{C}$ to $\mathrm{OD}_{600}$ of $\sim 0.3$, then maintained at $30{ }^{\circ} \mathrm{C}(\mathrm{B})$ or shifted to $45^{\circ} \mathrm{C}(\mathrm{C})$ for $60 \mathrm{~min}$ before dilution and growth rate measurement at 37 ${ }^{\circ} \mathrm{C}$ in flasks.

(D-I) Growth curves for WT and $\Delta 10 \mathrm{TA}$ strains grown at $37^{\circ} \mathrm{C}$ and left untreated (D) or treated with the indicated stress for one hour then washed and diluted into fresh media, as described in (A). For complete description of stress conditions, see Methods.

(J) Wild-type and $\triangle 10 T A$ strains labeled with YFP and CFP, respectively, were mixed at a 1:1 ratio. This mixture was propagated and treated with two cycles of chloramphenicol as depicted in the schematic. Samples were taken points indicated by numbers on left, and colony forming units (c.f.u.) of the two strains were determined. The resulting competitive index is graphed on the right where individual symbols indicate independent replicates; black bar represents mean. 
(K) Growth curve of WT and $\Delta 10 \mathrm{TA}$ strains grown at $44{ }^{\circ} \mathrm{C}$.

(L-M) Growth curve of WT and $\Delta 10 \mathrm{TA}$ strains grown to OD600 0.3, then treated with the indicated stress throughout growth measurements.

(N) Growth curves for WT and $\Delta 10 \mathrm{TA}$ strains grown at $37^{\circ} \mathrm{C}$ and treated with chloramphenicol for four hours, then washed and diluted into fresh media, as described in (A).

Figure 3. RNA-seq reveals no evidence of endoribonuclease toxin activity following stress.

(A) Example analysis for MazF cleavage site (yellow shaded box) found within the $r p l S$ transcript. Summed read counts for empty vector and MazF overexpression (upper panel) and wild-type or $\Delta$ 10TA following chloramphenicol treatments at indicated time points (second panel). The ratios of MazF / empty vector and WT / $\triangle 10 \mathrm{TA}$ in chloramphenicol are shown (third panel) with the slop of the ratio profiles below (fourth panel). The known MazF cleavage motif is indicated (red arrow).

(B-C) Ratio profiles (upper) and resulting ratio slopes (lower) are shown for YoeB cleavage site (yellow box) in the mtlD transcript (B) and MqsR cleavage site (yellow box) in the rluD transcript (C).

(D) Average of the slope maxima in the ratio profiles of coding regions corresponding to 'MazF targets' as defined in the text (red dot), compared to a distribution generated from sampling random sets of coding regions of the same size 10,000 times from the same sample (grey box and whiskers). The z-score calculated from this comparison is indicated (left). The same analysis was performed on the WT v. $\triangle 10 \mathrm{TA}$ in chloramphenicol data sets, comparing MazF targets to a distribution based on random samples.

(E) Z-scores resulting from the analysis described in (D), but for each of the nine TA systems indicated.

See also Figures S2 and S3.

Figure 4. Increases in TA transcription result from a relief of autoregulation.

(A) Antitoxin transcript levels were measured in untreated cells at $\mathrm{OD}_{600} \sim 0.3$ by qRT-PCR for the wild-type and corresponding DNA-binding mutant allele for the indicated antitoxin. Transcripts were normalized to the gyrA housekeeping gene. Data are the average of 3 biological replicates with error bars indicating the S.D. 
(B) Changes in antitoxin transcript levels in chloramphenicol-treated compared to untreated cells as measured by qRT-PCR. Mutations abrogating DNA-binding by each antitoxin were introduced on the chromosome. Data are the average of 3 biological replicates with error bars indicating the S.D.

(C) Changes in antitoxin levels for chloramphenicol-treated cells compared with untreated cells of the wild-type strain or the indicated protease deletion mutant. Transcript levels were quantified by qRT-PCR. Data are the average of 2 biological replicates with error bars representing the S.D.

\section{Figure 5. ppGpp is not required for TA transcription.}

(A) Previous model for ppGpp-mediated activation of TA systems proposed by Maisonneuve et. al. (2013). Amino-acid starvation induces ppGpp, which inhibits polyphosphatase (Ppx), allowing polyphosphate kinase (Ppk) to synthesize polyphosphate, which then stimulates the Lon protease to degrade antitoxins and liberate toxins.

(B) Anion exchange chromatography traces for nucleotide extracts from cells harvested 30 min after the indicated treatment. Arrow indicates peak corresponding to ppGpp.

(C) Quantification of ppGpp levels in cells after the indicated treatments. Data points are the average of 3 biological replicates with error bars indicating S.D.

(D-E) Change in antitoxin transcript levels in cells treated with either SHX (D) or chloramphenicol (E) for 30 minutes compared to untreated cells. Transcript levels were quantified by qRT-PCR. Errors bars represent S.D. and data are the average of 3 biological replicates.

(F-G) Antitoxin degradation as measured by pulse-chase analysis. Strains bearing a medium-copy plasmid harboring a TA system under the control of its native promoter, pBR322-mqsRA (F) or pBR322-his $\sigma_{-}$-yefM-yoeB $(\mathrm{G})$, were grown to $\mathrm{OD}_{600} \sim 0.3$, then pulsed for 10 min with radiolabeled $\mathrm{S}^{35}$ cysteine and methionine, and chloramphenicol or SHX added at the same time as the unlabeled cysteine and methionine used to chase. Cell pellets were subsequently lysed and antitoxin was immunoprecipitated and analyzed by autoradiography. Representative gels are shown above with a quantification of at least 2 biological replicates shown below. Error bars indicate S.D.

\section{Figure 6. Autoregulation maintains antitoxin homeostasis despite changes in degradation.}

(A) Antitoxin degradation rate of His6-YefM (A) measured by pulse-chase analysis in cells grown at $30{ }^{\circ} \mathrm{C}$ and then either kept at $30^{\circ} \mathrm{C}$ (untreated) or shifted to $45^{\circ} \mathrm{C}$ (heat shock). 
(B) Cellular antitoxin levels were measured for His6-YefM by steady-state radiolabeling followed by immunoprecipitation and autoradiography using the same growth conditions as in panels A.

(C) Model for transcriptional induction of yefM-yoeB following a stress like heat shock. Stress leads to increased degradation of YefM antitoxin, particularly free antitoxin. As the ratio of toxin:antitoxin increases, YefM antitoxin cannot efficiently bind the yefM-yoeB promoter, leading to sustained transcriptional induction.

(D) Degradation rates measured by pulse-chase analysis for His6-YefM expressed with its cognate toxin, YoeB (green) or by itself (orange).

(E) Antitoxin degradation rate of His6-YefM* measured as described in panel A.

(F) Cellular antitoxin levels of His6-YefM* measured as described in panel B.

(G) Antitoxin degradation rate of MqsA measured as described in panel A.

(H) Cellular antitoxin levels of MqsA measured from wild-type cells by western blot.

(I) Degradation rates measured by pulse-chase analysis for MqsA expressed with its cognate toxin, MqsR (green) or by itself (orange).

(J) Antitoxin degradation rate of MqsA* measured as described in panel A.

(K) Cellular antitoxin levels of MqsA* measured as described in panel H.See also Fig. S4.

Figure 7. Chromosomal TA systems are not induced by an inhibition of new synthesis as with the plasmid-borne system CcdAB.

(A) Schematic for growth experiments in panels B-D. Strains were grown to $\mathrm{OD}_{600} \sim 0.3$ in flasks, then split into 2 flasks, one of which was induced with $0.2 \%$ arabinose for $15 \mathrm{~min}$. After washing, cells were released into repressing conditions (M9 medium containing glucose) at a 100x dilution and growth was measured on a plate reader.

(B-D) Growth curves resulting from experiment described in panel A for (B) attL::P ara-ccdAB, (C) $\Delta y e f M-y o e B$ attL $:: P_{\text {ara }}-y e f M-y o e B$, and (D) $\Delta m q s R A$ attL $:: P_{\text {ara }}-m q s R A$. 


\section{Supplemental Figure Legends}

Figure S1. TA systems are transcriptionally activated by diverse stresses.

Difference in antitoxin transcript levels between cells exposed to the indicated stress for 30 minutes compared to untreated cells as measured by qRT-PCR corresponding to Figure 1D.

Data presented are the average of 3 biological replicates errors bar represent S.D.

Figure S2. Transcripts targeted by toxins are not cleaved after chloramphenicol treatment.

(A-I) Examples of transcripts that show signatures of cleavage following toxin overexpression. Ratios of $+/$ - toxin and chloramphenicol-treated wild type / $\Delta 10 \mathrm{TA}$ (upper panel) and corresponding slopes (lower panel) for each of the nine toxins examined.

See also Figure 3.

Figure S3. No evidence of toxin cleavage following chloramphenicol treatment.

(A) Comparison of of the shape of ratio plots of $+\mathrm{MazF} /$ empty vector for $r p l S$ transcript containing a MazF cleavage site at high, medium, and low MazF expression levels (upper 3 panels) and for wild type / $\triangle 10 \mathrm{TA}$ in chloramphenicol treatment samples (fourth panel). Lower panel is an overlay of all five ratio profiles plotted on the same scale.

(B) YhaV regions containing the highest cleavage ratio slopes corresponding to analysis described in Fig. 3E. Chloramphenicol-treated samples are compared to $\mathrm{YhaV}$ overexpression data.

(C) Z-scores generated from comparing the average of the minima detected in the transcripts with the lowest + - - toxin ratios (lowest 5\%, from toxin overexpression datasets) to the average of the minima from a randomly selected set of transcripts (sampled 10,000 times) in the chloramphenicol RNA-seq datasets. A negative z-score indicates a significant amount of toxin activity; positive zscores may result from transcript stabilization.

See also Figure 3.

Figure S4. Chloramphenicol-induced changes in transcription require the Lon protease. Difference in antitoxin levels of chloramphenicol-treated compared with untreated cells of the indicated protease deletion mutants for $m q s R A(\mathrm{~A})$, yefM-yoeB (B), and relBE (C). Transcript levels were quantified by qRT-PCR and correspond to Fig. 4B. 
Data presented are the average of 2 biological replicates and error bars represent S.D.

See also Figure 4.

Figure S5. Autoregulation maintains antitoxin homeostasis despite changes in degradation.

(A-B) Representative phosphorimages of $\mathrm{His}_{6}$-YefM degradation (A) or cellular antitoxin levels (B) in cells grown at $30{ }^{\circ} \mathrm{C}$ and then either kept at $30{ }^{\circ} \mathrm{C}$ (untreated) or shifted to $45{ }^{\circ} \mathrm{C}$ (heat shock). Degradation was measured by pulse-chase and immunoprecipitation while cellular levels were measured by steady-state radiolabeling followed by immunoprecipitation and autoradiography.

(C) Representative phosphorimages of His $_{6}$-YefM degradation following co-expression with its cognate toxin, YoeB (upper), or expression by itself (lower).

(D-E) Same as panels A-B but for ${ }_{\text {His6-YefM* }}$.

(F-G) Representative phosphorimages of MqsA degradation measured by pulse chase ((F) or Western blots of cellular MqsA levels (G) under growth conditions described in (A).

(H) Model for MqsA protein levels following stress. MqsA cannot bind both DNA and MqsR, therefore at higher degradation rates as toxin:antitoxin levels increase, the promoter is derepressed, leading to transcriptional activation.

(I) Representative phosphorimages of MqsA degradation following co-expression with its cognate toxin, MqsR (upper), or expression by itself (lower).

(J-K) Same as panels F-G but for MqsA*.

See also Figure 6. 


\section{Methods}

\section{Strains and growth conditions}

All primers and strains used in this study are listed in Tables S1 and S2, respectively. E. coli was cultivated at $37{ }^{\circ} \mathrm{C}$ in $\mathrm{M} 9$-glucose $\left(6.4 \mathrm{~g} / \mathrm{L} \mathrm{Na}_{2} \mathrm{HPO}_{4}-7 \mathrm{H}_{2} \mathrm{O}, 1.5 \mathrm{~g} / \mathrm{L} \mathrm{KH}_{2} \mathrm{PO}_{4}, 0.25 \mathrm{~g} / \mathrm{L} \mathrm{NaCl}, 0.5\right.$ $\mathrm{g} / \mathrm{L} \mathrm{NH}_{4} \mathrm{Cl}$ medium supplemented with $0.1 \%$ casamino acids, $0.4 \%$ glucose, $2 \mathrm{mM} \mathrm{MgSO}_{4}$, and $0.1 \mathrm{mM} \mathrm{CaCl}_{2}$ ) for all experiments, except when otherwise indicated. Routine cultivation for strain construction was in Luria broth (LB). Media for selection or plasmid maintenance were supplemented with carbenicillin $(100 \mu \mathrm{g} / \mathrm{mL})$, chloramphenicol $(20 \mu \mathrm{g} / \mathrm{mL})$, or kanamycin $(30$ $\mu \mathrm{g} / \mathrm{ml})$ as necessary unless otherwise indicated.

\section{Plasmid construction}

TA systems were inserted by Gibson assembly into pBR322 amplified without the tetracycline promoter using primers ML1 and ML2. The region of the chromosome encoding each TA locus, including approximately $200 \mathrm{bp}$ upstream, was amplified with primers ML3 and ML4 (yefMyoeB), ML5 and ML6 (mqsRA), and ML7 and ML8 (relBE) with 40 bp ends homologous to pBR322. Mutations previously reported to abrogate DNA binding were introduced into pBR322$m q s R A, \mathrm{pBR} 322-r e l B E$, and pBR322-yefM-yoeB by site-directed mutagenesis using inverse PCR with primers ML15 and ML16 (yefM), ML17 and ML18 (mqsA), and ML19 and ML20 (relB) resulting in pBR322-yefM*-yoeB, pBR322-mqsRA(N97A), pBR322-relB*E.

pBR322-His 6 -yefM-yoeB and pBR322-His $6-$ yef $^{*}$-yoeB were constructed by site-directed mutagenesis with inverse PCR using primers ML23 and ML24 using pBR322-yefM-yoeB and pBR322-yef $M^{*}$-yoeB as templates, respectively. pBR322-His 6 -yefM was constructed by inverse PCR using primers ML25 and ML26 with pBR322-His 6 -yefM-yoeB as the template. pKVS45$m q s R A$ and pKVS45-mqs $A$ were constructed using restriction based cloning. MqsRA or MqsA were amplified from MG1655 using primers ML27 and ML28 or ML28 and ML29, respectively, digested with SacI and HindIII, and ligated to pKVS45 digested with the same enzymes.

\section{Strain construction}

Fluorescently labeled wild-type and $\Delta 10 \mathrm{TA}$ strains were generated by transduction of $g a l K:: P_{l a c}{ }^{-}$ $c f p$ and $g a l K:: P_{l a c}-y f p$ (Elowitz et. al. 2002). The DNA-binding mutants were constructed by allelic exchange, wherein the TA locus was first replaced with a sacB-neoR cassette from pIB279 
amplified with primer sets ML9 and ML10 (yefM-yoeB), ML11 and ML12 (mqsRA), and ML13 and ML14 (relBE) (Blomfield et al., 1991). The DNA-binding mutant alleles were then amplified from $\mathrm{pBR} 322-r e l B^{*} E, \mathrm{pBR} 322-m q s R A^{*}$, and $\mathrm{pBR} 322-y e f M^{*}$-yoeB described above with homologous ends to the flanking region. Both the counterselectable cassette and subsequent DNAbinding mutant allele were inserted using lambda Red recombinase, either selected on kanamycin or counterselected on 5\% sucrose plates as previously described (Datsenko and Wanner, 2000). Mutations were verified by PCR amplification and sequencing.

To make protease deletions, deletion strains were obtained from the Keio collection and transduced into MG1655 with P1 phage. Kanamycin resistance cassettes were removed using pFLP1 excision as previously described (Datsenko and Wanner, 2000). The $\Delta p p k \Delta p p x$ strain was constructed by lambda Red to replace the locus with a kanamycin resistance cassette amplified from pKD4 using primers ML21 and ML22 with homology flanking the $p p k p p x$ region. The kanamycin resistance cassette was removed with pFLP1 excision.

Arabinose-inducible TA systems were created by in-frame deletion of the TA systems. The neoR$s a c B$ cassette replacement strains described above were transformed with corresponding deletion oligos ML36 (yefM-yoeB) and ML37 (mqsRA) and deletions were counterselected on sucrose plates. TA open reading frames were then inserted downstream of the arabinose promoter of the pAH150 plasmid (Haldimann and Wanner, 2001) by Gibson assembly. TA systems were amplified with primers ML40 and ML41 ( $c c d A B$, amplified from the F plasmid); ML42 and ML42 (yefMyoeB), and ML43 and ML44 (mqsRA) and pAH150 linearized with primers ML38 and ML39. CRIM insertion was then performed as previously described using helper plasmid pInt-ts with selection on low kanamycin $(6 \mu \mathrm{g} / \mathrm{mL})$ to prevent multiple insertion events (Haldimann and Wanner, 2001). Single insertions were confirmed by PCR as described.

\section{Stress conditions}

For all stress conditions used in qRT-PCR, pulse-chase, immunoblots, and growth experiments, stresses were applied as follows: chloramphenicol at $75 \mu \mathrm{g} / \mathrm{mL}$, SHX at $100 \mu \mathrm{g} / \mathrm{mL}$, trimethoprim at $25 \mu \mathrm{g} / \mathrm{mL}, \mathrm{H}_{2} \mathrm{O}_{2}$ (Sigma) at $0.021 \% \mathrm{vol} / \mathrm{vol}(7 \mathrm{mM})$, carbenicillin at $25 \mu \mathrm{g} / \mathrm{mL}$. For low $\mathrm{pH}$ experiments, cells were washed into M9-glucose adjusted to $\mathrm{pH}$ of 4 for the duration of the stress treatment. For all heat shock experiments, cells were grown at $30{ }^{\circ} \mathrm{C}$ prior to heat shock, then either kept at $30{ }^{\circ} \mathrm{C}$ (untreated) or shifted to $45^{\circ} \mathrm{C}$ (heat shock). 


\section{qRT-PCR experiments}

Cells were harvested by adding $900 \mu \mathrm{L}$ bacterial culture to $100 \mu \mathrm{L}$ stop solution ( $5 \%$ phenol, $95 \%$ ethanol $\mathrm{v} / \mathrm{v}$ ) on ice. RNA extractions, reverse transcription, and qRT-PCR were performed as described previously (Culviner and Laub, 2018) with the primers listed in Table S1. Briefly, RNA was reverse-transcribed with first-strand synthesis kit (Invitrogen) using random primers. cDNA was diluted 1:15. Standard curves made by combining and diluting cDNA from each set of cDNAs were run for all primers on each plate. Reactions were prepared using 2X SYBR Fast Mastermix (Roche) and run on a Light Cycler 480 II Real-time PCR Machine at the MIT BioMicro Center in technical duplicates. Samples were analyzed using the standard-curve method and transcripts of interest were normalized to the gyrA housekeeping gene. All qRT-PCR data reported are the average of biological triplicates, with the exception of the protease deletion strains, which are the average of biological duplicates.

\section{Growth experiments}

For growth experiments, the wild type used was the parental strain to the $\Delta 10 \mathrm{TA}$ strain obtained from L. Van Melderen (Goormaghtigh et al., 2018). Cells were grown to $\mathrm{OD}_{600} \sim 0.3$ and then stresses were applied as described above. After the indicated stress treatment, cells were washed $2 \mathrm{x}$ in an equal volume of media. Cells were diluted to $\mathrm{OD}_{600}$ of 0.01 in M9-glucose. Growth was measured in 96-well plates at 15 min intervals $(180 \mu \mathrm{L}$ culture overlaid with $70 \mu \mathrm{L}$ mineral oil) with orbital shaking at $37^{\circ} \mathrm{C}$ on a plate reader (Biotek). Representative data presented are the result of 6-12 plate replicates and were replicated independently at least 2 times. For growth measurements during heat shock, cells were diluted to $\mathrm{OD}_{600} \sim 0.01$ and then growth curves were performed on a plate reader set either to $30^{\circ} \mathrm{C}$ or $44^{\circ} \mathrm{C}$. For the competition experiments, overnight cultures of fluorescently labeled wild-type and $\Delta 10 \mathrm{TA}$ were mixed at a 1:1 ratio, then treated to chloramphenicol as described for growth curve experiments. After the first chloramphenicol treatment, cultures were washed and back-diluted in M9-glucose to an $\mathrm{OD}_{600}$ of 0.05 and allowed to reach $\mathrm{OD}_{600} \sim 0.3$ before the second chloramphenicol treatment, second wash, and final growth step. Samples were taken at indicated time points, including after overnight growth. Each sample was serially diluted and plated on non-selective plates. Plates were scanned on a Typhoon imager and CFP vs YFP colonies determined using the CY3 fluorescent channel. The experiment was performed with three biological replicates. 


\section{RNA-seq and analysis}

Cells were collected and RNA harvested as described above. RNA-seq libraries were prepared as described previously (Culviner and Laub, 2018). Briefly, rRNA was removed with a RiboZero kit (Illumina) per manufacturer instructions. RNA was fragmented with RNA Fragmentation reagents (Invitrogen). Fragmentation reagent was added, then samples were incubated at $70{ }^{\circ} \mathrm{C}$ for 8 min before reactions were stopped. Fragmented RNA was ethanol precipitated, then reversetranscribed using random primers (Invitrogen First Strand Kit and Random Primers). Second strand synthesis was subsequently performed (Invitrogen Second Strand Synthesis kit) and RNA digested with Rnase H (NEB). Paired-end sequencing was performed on an Illumina NextSeq 500 at the MIT BioMicroCenter. Data were uploaded to NCBI Geo (accession number GSE141320).

\section{RNA-Seq Analysis}

RNA-Seq read mapping and calculation of + toxin : empty vector ratios were conducted as described previously (Culviner and Laub, 2018). Slopes were calculated by a least-squares fit of the 30 nucleotides to the $5^{\prime}$ of a given position. To systematically search for cleavage across the transcriptome, we first identified coding genes that had at least 64 counts at all positions across the coding region in the empty vector sample and at least 1 count in the expressed toxin sample. Toxin-antitoxin genes as well as the phase-variable $f l u$ gene were excluded from this analysis regardless of their expression level. For slope-based identification of cleavage targets, the maximum slope (calculated as described above) was calculated for each transcript. The transcripts with the top $5 \%$ of maximum slopes were classified as toxin targets. To determine if cleavage was occurring in the presence of chloramphenicol, we took the ratio profiles for wild type v. $\Delta 10 \mathrm{TA}$ in chloramphenicol and calculated the mean of the maximum slopes for the defined set of toxin targets and compared this value to the mean of the maximum slopes for randomly selected transcripts. To do this, we sampled sets of randomly chosen genes (the same number as the number of toxin targets) 10,000 times and calculated the mean of their maximum slopes. In this way, we generated a distribution of the expected value of mean maximum slopes. These distributions are shown as box and whisker plots displaying the median, lower and upper quartiles, and quartile \pm 1.5 interquartile range (Fig. 3D). The mean maximum slope of toxin targets was compared to these distributions to calculate a z-score. The above analysis was also modified to detect cleavage by + toxin : empty vector ratios. In this case, the bottom $20 \%$ of transcript minimum ratios were 
classified as toxin targets. The rest of the analysis was conducted as above, but minimum ratios replaced maximum slopes.

\section{ppGpp measurements}

Cells were grown to $\mathrm{OD}_{600}$ of $0.2-0.3$, then treated with either chloramphenicol or $\mathrm{SHX}$ as described, and harvested and quantified as described previously (Kraemer et al., 2019). Briefly, cells were vacuum filtered onto a nitrocellulose membrane and immediately exposed to cold lysis solvent ( $40 \%$ methanol, $40 \%$ acetonitrile, $20 \%$ water), removed from the membrane by sonication, and flash frozen. Volumes were adjusted based on $\mathrm{OD}_{600}$ of the culture at the time of harvest, and ppGpp was quantified from the resulting nucleotide extracts by anion-exchange chromatography (MonoQ 5/50 column) and concentration calculated based on a standard curve. Data presented are the result of biological triplicates.

\section{Pulse chase experiments}

Cells were grown in M9-glucose to $\mathrm{OD}_{600} \sim 0.3$. Cultures were labeled with $80 \mu \mathrm{Ci} / \mathrm{mL}\left[{ }^{35} \mathrm{~S}\right]$ EasyTag $^{\text {TM }}$ EXPRESS $^{35}$ S Protein Labeling Mix (Perkin Elmer) for $10 \mathrm{~min}$, then chased with $2 \mathrm{mM}$ cysteine and methionine, and the indicated stress applied. For all time points, $1 \mathrm{~mL}$ of cells were pelleted and flash frozen in liquid nitrogen. Pellets were resuspended in $500 \mu \mathrm{L}$ lysis buffer (PBS supplemented with $0.05 \%$ Tween-20, $1 \mu \mathrm{L} / \mathrm{mL}$ benzonase, $1 \mu \mathrm{L} / \mathrm{mL}$ ReadyLyse (Novagen), and cOmplete protease inhibitor cocktail (Sigma)). Cells were lysed by additional freeze/thaw cycles, cell debris was removed by centrifugation, and proteins were immunoprecipitated with Protein A Dynabeads (ThermoFisher) conjugated to the relevant antibody (anti-MqsRA; anti-His for His $6^{-}$ YefM). Samples were resolved by $4-20 \%$ SDS-PAGE, and then the gel was dried, exposed to Phosphorimaging screens for 1-10 days as needed, and scanned with a Typhoon scanner (GE Healthcare). For the experiment comparing MqsA degradation following expression alone or coexpression with its cognate toxin, the toxin ribosome-binding site was changed to that of the antitoxin to increase toxin expression levels. All pulse-chase data presented are the average of at least two independent biological replicates.

\section{Western blots}

Cells were harvested by centrifugation and flash frozen in liquid nitrogen. Pellets were resuspended in 1x Laemmli buffer and analyzed by SDS-PAGE. Anti-MqsRA antibody was 
generated using $\mathrm{His}_{6}$-MqsRA complex (Covance) and used unpurified at 1:3000. Anti-His 6 antibody was used at 1:5000 (Thermo Fisher); and purified anti-Lon antibody was used at 1:5000 (kind gift from T. Baker and R. Sauer). SuperSignal West Femto Maximum Sensitivity Substrate (ThermoFisher) was used to develop the blots, which were visualized with a FluorChem R Imager (ProteinSimple). Because high amounts of lysate were loaded onto gels to allow for antitoxin detection, typical loading controls were outside the linear range. Loading normalization was instead done by Coomassie staining membranes after Western blotting. All Western blot data presented are the average of three independent biological replicates.

\section{Arabinose promoter shut-off experiments}

Cells were grown to $\mathrm{OD}_{600} \sim 0.3$ in M9 media prepared as described previously, but supplemented with $0.4 \%$ glycerol instead of glucose. Cells were then induced with $0.4 \% \mathrm{~L}$-arabinose for $15 \mathrm{~min}$ after which they were washed in M9-glucose, and diluted to $\mathrm{OD}_{600}$ of 0.01 in M9-glucose. Growth curves were generated on a plate reader as described above. 


\section{References}

Ahn, B.-E., Baker, T.A., 2016. Oxidization without substrate unfolding triggers proteolysis of the peroxide-sensor, PerR. Proc Natl Acad Sci USA 113, E23-E31. doi:10.1073/pnas.1522687112

Aizenman, E., Engelberg-Kulka, H., Glaser, G., 1996. An Escherichia coli chromosomal "addiction module" regulated by guanosine [corrected] 3“,5-”bispyrophosphate: a model for programmed bacterial cell death. Proc Natl Acad Sci USA 93, 6059-6063.

Bailey, S.E.S., Hayes, F., 2009. Influence of Operator Site Geometry on Transcriptional Control by the YefM-YoeB Toxin-Antitoxin Complex. J. Bacteriol. 191, 762-772. doi:10.1128/JB.01331-08

Blomfield, I.C., Vaughn, V., Rest, R.F., Eisenstein, B.I., 1991. Allelic exchange in Escherichia coli using the Bacillus subtilis sacB gene and a temperature-sensitive pSC101 replicon. Molecular Microbiology 5, 1447-1457. doi:10.1111/j.1365-2958.1991.tb00791.x

Brown, B.L., Lord, D.M., Grigoriu, S., Peti, W., Page, R., 2013. The Escherichia coli toxin MqsR destabilizes the transcriptional repression complex formed between the antitoxin MqsA and the mqsRA operon promoter. J. Biol. Chem. 288, 1286-1294. doi:10.1074/jbc.M112.421008

Brown, B.L., Wood, T.K., Peti, W., Page, R., 2011. Structure of the Escherichia coli antitoxin MqsA (YgiT/b3021) bound to its gene promoter reveals extensive domain rearrangements and the specificity of transcriptional regulation. J. Biol. Chem. 286, 2285-2296. doi:10.1074/jbc.M110.172643

Cherny, I., 2005. The YoeB Toxin Is a Folded Protein That Forms a Physical Complex with the Unfolded YefM Antitoxin: IMPLICATIONS FOR A STRUCTURAL-BASED DIFFERENTIAL STABILITY OF TOXIN-ANTITOXIN SYSTEMS. Journal of Biological Chemistry 280, 30063-30072. doi:10.1074/jbc.M506220200

Christensen, S.K., Gerdes, K., 2004. Delayed-relaxed response explained by hyperactivation of RelE. Molecular Microbiology 53, 587-597. doi:10.1111/j.1365-2958.2004.04127.x

Christensen, S.K., Mikkelsen, M., Pedersen, K., Gerdes, K., 2001. RelE, a global inhibitor of translation, is activated during nutritional stress. Proc Natl Acad Sci USA 98, 14328-14333. doi:10.1073/pnas.251327898

Christensen, S.K., Pedersen, K., Hansen, F.G., Gerdes, K., 2003. Toxin-antitoxin Loci as Stressresponse-elements: ChpAK/MazF and ChpBK Cleave Translated RNAs and are Counteracted by tmRNA. J. Mol. Biol. 332, 809-819. doi:10.1016/S0022-2836(03)00922-7

Christensen-Dalsgaard, M., Jørgensen, M.G., Gerdes, K., 2010. Three new RelE-homologous mRNA interferases of Escherichia coli differentially induced by environmental stresses. Molecular Microbiology 75, 333-348. doi:10.1111/j.1365-2958.2009.06969.x

Culviner, P.H., Laub, M.T., 2018. Global Analysis of the E. coli Toxin MazF Reveals Widespread Cleavage of mRNA and the Inhibition of rRNA Maturation and Ribosome Biogenesis. Mol. Cell 70, 868-880.e10. doi:10.1016/j.molcel.2018.04.026

Datsenko, K.A., Wanner, B.L., 2000. One-step inactivation of chromosomal genes in Escherichia coli K-12 using PCR products. Proc Natl Acad Sci USA 97, 6640-6645. doi:10.1073/pnas.120163297

Dervyn, E., Canceill, D., Huisman, O., 1990. Saturation and specificity of the Lon protease of Escherichia coli. J. Bacteriol. 172, 7098-7103. doi:10.1128/jb.172.12.7098-7103.1990

Gerdes, K., Maisonneuve, E., 2012. Bacterial persistence and toxin-antitoxin loci. Annu. Rev. 
Microbiol. 66, 103-123. doi:10.1146/annurev-micro-092611-150159

Goeders, N., Dreze, P.L., Van Melderen, L., 2013. Relaxed Cleavage Specificity within the RelE Toxin Family. J. Bacteriol. 195, 2541-2549. doi:10.1128/JB.02266-12

Goormaghtigh, F., Fraikin, N., Putrinš, M., Hallaert, T., Hauryliuk, V., Garcia-Pino, A., Sjödin, A., Kasvandik, S., Udekwu, K., Tenson, T., Kaldalu, N., Van Melderen, L., 2018.

Reassessing the Role of Type II Toxin-Antitoxin Systems in Formation of Escherichia coli Type II Persister Cells. mBio 9, 1479. doi:10.1128/mBio.00640-18

Haldimann, A., Wanner, B.L., 2001. Conditional-replication, integration, excision, and retrieval plasmid-host systems for gene structure-function studies of bacteria. J. Bacteriol. 183, 63846393. doi:10.1128/JB.183.21.6384-6393.2001

Harms, A., Fino, C., Sørensen, M.A., Semsey, S., Gerdes, K., 2017. Prophages and Growth Dynamics Confound Experimental Results with Antibiotic-Tolerant Persister Cells. mBio 8, e01964-17-18. doi:10.1128/mBio.01964-17

Harms, A., Maisonneuve, E., Gerdes, K., 2016. Mechanisms of bacterial persistence during stress and antibiotic exposure. Science 354, aaf4268-aaf4268. doi:10.1126/science.aaf4268

Harrison, J.J., Wade, W.D., Akierman, S., Vacchi-Suzzi, C., Stremick, C.A., Turner, R.J., Ceri, H., 2009. The Chromosomal Toxin Gene yafQ Is a Determinant of Multidrug Tolerance for Escherichia coli Growing in a Biofilm. Antimicrob. Agents Chemother. 53, 2253-2258. doi:10.1128/AAC.00043-09

Hazan, R., Sat, B., Engelberg-Kulka, H., 2004. Escherichia coli mazEF-Mediated Cell Death Is Triggered by Various Stressful Conditions. J. Bacteriol. 186, 3663-3669. doi:10.1128/JB.186.11.3663-3669.2004

Helaine, S., Cheverton, A.M., Watson, K.G., Faure, L.M., Matthews, S.A., Holden, D.W., 2014. Internalization of Salmonella by macrophages induces formation of nonreplicating persisters. Science 343, 204-208. doi:10.1126/science.1244705

Jaffé, A., Ogura, T., Hiraga, S., 1985. Effects of the ccd function of the F plasmid on bacterial growth. J. Bacteriol. 163, 841-849.

Janssen, B.D., Garza-Sánchez, F., Hayes, C.S., 2015. YoeB toxin is activated during thermal stress. MicrobiologyOpen 4, 682-697. doi:10.1002/mbo3.272

Jonas, K., Liu, J., Chien, P., Laub, M.T., 2013. Proteotoxic Stress Induces a Cell-Cycle Arrest by Stimulating Lon to Degrade the Replication Initiator DnaA. Cell 154, 623-636. doi:10.1016/j.cell.2013.06.034

Kaplan, S., Atherly, A.G., Barrett, A., 1973. Synthesis of Stable RNA in Stringent Escherichia coli Cells in the Absence of Charged Transfer RNA. Proc Natl Acad Sci USA 70, 689-692. doi:10.1073/pnas.70.3.689

Kedzierska, B., Lian, L.Y., Hayes, F., 2006. Toxin-antitoxin regulation: bimodal interaction of YefM-YoeB with paired DNA palindromes exerts transcriptional autorepression. Nucleic Acids Res 35, 325-339. doi:10.1093/nar/gk11028

Koga, M., Otsuka, Y., Lemire, S., Yonesaki, T., 2011. Escherichia coli rnlA and rnlB Compose a Novel Toxin-Antitoxin System. Genetics 187, 123-130. doi:10.1534/genetics.110.121798

Kolodkin-Gal, I., Engelberg-Kulka, H., 2006. Induction of Escherichia coli Chromosomal mazEF by Stressful Conditions Causes an Irreversible Loss of Viability. J. Bacteriol. 188, 3420-3423. doi:10.1128/JB.188.9.3420-3423.2006

Kraemer, J.A., Sanderlin, A.G., Laub, M.T., 2019. The Stringent Response Inhibits DNA Replication Initiation in E. coli by Modulating Supercoiling of oriC. mBio 10, 822. doi:10.1128/mBio.01330-19 
Kuroda, A., Nomura, K., Ohtomo, R., Kato, J., Ikeda, T., Takiguchi, N., Ohtake, H., Kornberg, A., 2001. Role of inorganic polyphosphate in promoting ribosomal protein degradation by the Lon protease in E. coli. Science 293, 705-708. doi:10.1126/science.1061315

Li, G.-W., Burkhardt, D., Gross, C., Weissman, J.S., 2014. Quantifying Absolute Protein Synthesis Rates Reveals Principles Underlying Allocation of Cellular Resources. Cell 157, 624-635. doi:10.1016/j.cell.2014.02.033

Magnuson, R.D., 2007. Hypothetical functions of toxin-antitoxin systems. J. Bacteriol. 189, 6089-6092. doi:10.1128/JB.00958-07

Mahmoud, S.A., Chien, P., 2018. Regulated Proteolysis in Bacteria. Annu. Rev. Biochem. 87, 677-696. doi:10.1146/annurev-biochem-062917-012848

Maisonneuve, E., Castro-Camargo, M., Gerdes, K., 2013. (p)ppGpp Controls Bacterial Persistence by Stochastic Induction of Toxin-Antitoxin Activity. Cell 154, 1140-1150. doi:10.1016/j.cell.2013.07.048

Maisonneuve, E., Gerdes, K., 2014. Molecular mechanisms underlying bacterial persisters. Cell 157, 539-548. doi:10.1016/j.cell.2014.02.050

Maisonneuve, E., Shakespeare, L.J., Jørgensen, M.G., Gerdes, K., 2011. Bacterial persistence by RNA endonucleases. Proc. Natl. Acad. Sci. U.S.A. 108, 13206-13211. doi:10.1073/pnas.1100186108

Mizusawa, S., Gottesman, S., 1983. Protein degradation in Escherichia coli: the lon gene controls the stability of sulA protein. Proc Natl Acad Sci USA 80, 358-362. doi:10.1073/pnas.80.2.358

Muthuramalingam, M., White, J., Bourne, C., 2016. Toxin-Antitoxin Modules Are Pliable Switches Activated by Multiple Protease Pathways. Toxins 8, 214-16. doi:10.3390/toxins 8070214

Nigam, A., Ziv, T., Oron-Gottesman, A., Engelberg-Kulka, H., 2019. Stress-Induced MazFMediated Proteins in Escherichia coli. mBio 10, 6059-9. doi:10.1128/mBio.00340-19

Ogura, T., Inoue, K., Tatsuta, T., Suzaki, T., Karata, K., Young, K., Su, L.H., Fierke, C.A., Jackman, J.E., Raetz, C.R.H., Coleman, J., Tomoyasu, T., Matsuzawa, H., 1999. Balanced biosynthesis of major membrane components through regulated degradation of the committed enzyme of lipid A biosynthesis by the AAA protease FtsH (HflB) in Escherichia coli. Molecular Microbiology 31, 833-844. doi:10.1046/j.1365-2958.1999.01221.x

Overgaard, M., Borch, J., Gerdes, K., 2009. RelB and RelE of Escherichia coli Form a Tight Complex That Represses Transcription via the Ribbon-Helix-Helix Motif in RelB. J. Mol. Biol. 394, 183-196. doi:10.1016/j.jmb.2009.09.006

Overgaard, M., Borch, J., Jørgensen, M.G., Gerdes, K., 2008. Messenger RNA interferase RelE controls relBE transcription by conditional cooperativity. Molecular Microbiology 69, 841857. doi:10.1111/j.1365-2958.2008.06313.x

Pruteanu, M., Neher, S.B., Baker, T.A., 2007. Ligand-Controlled Proteolysis of the Escherichia coli Transcriptional Regulator ZntR. J. Bacteriol. 189, 3017-3025. doi:10.1128/JB.01531-06

Prysak, M.H., Mozdzierz, C.J., Cook, A.M., Zhu, L., Zhang, Y., Inouye, M., Woychik, N.A., 2009. Bacterial toxin YafQ is an endoribonuclease that associates with the ribosome and blocks translation elongation through sequence-specific and frame-dependent mRNA cleavage. Molecular Microbiology 71, 1071-1087. doi:10.1111/j.1365-2958.2008.06572.x

Puri, N., Karzai, A.W., 2017. HspQ Functions as a Unique Specificity-Enhancing Factor for the AAA+ Lon Protease. Mol. Cell 66, 672-683.e4. doi:10.1016/j.molcel.2017.05.016

Ronneau, S., Helaine, S., 2019. Clarifying the Link between Toxin-Antitoxin Modules and 
Bacterial Persistence. J. Mol. Biol. \#startpage\#. doi:10.1016/j.jmb.2019.03.019

Salmon, M.A., Van Melderen, L., Bernard, P., Couturier, M., 1994. The antidote and autoregulatory functions of the F plasmid CcdA protein: a genetic and biochemical survey. Molec. Gen. Genet. 244, 530-538. doi:10.1007/BF00583904

Shah, I.M., Wolf, R.E., 2006. Inhibition of Lon-dependent degradation of the Escherichia coli transcription activator SoxS by interaction with "soxbox" DNA or RNA polymerase. Molecular Microbiology 60, 199-208. doi:10.1111/j.1365-2958.2006.05086.x

Shan, Y., Brown Gandt, A., Rowe, S.E., Deisinger, J.P., Conlon, B.P., Lewis, K., 2017. ATPDependent Persister Formation in Escherichia coli. mBio 8, e2267-16-14. doi:10.1128/mBio.02267-16

Sokawa, J., Sokawa, Y., 1978. Relaxation effect of chloramphenicol on the stringent control in Escherichia coli. J. Biochem. 83, 1699-1705. doi:10.1093/oxfordjournals.jbchem.a132083

Tsilibaris, V., Maenhaut-Michel, G., Mine, N., Van Melderen, L., 2007. What is the benefit to Escherichia coli of having multiple toxin-antitoxin systems in its genome? J. Bacteriol. 189, 6101-6108. doi:10.1128/JB.00527-07

Van Melderen, L., Aertsen, A., 2009. Regulation and quality control by Lon-dependent proteolysis. Research in Microbiologoy 160, 645-651. doi:10.1016/j.resmic.2009.08.021

Van Melderen, L., Bernard, P., Couturier, M., 1994. Lon-dependent proteolysis of CcdA is the key control for activation of CcdB in plasmid-free segregant bacteria. Molecular Microbiology 11, 1151-1157.

Van Melderen, L., Wood, T.K., 2017. Commentary: What Is the Link between Stringent Response, Endoribonuclease Encoding Type II Toxin-Antitoxin Systems and Persistence? Front. Microbiol. 8, R81-3. doi:10.3389/fmicb.2017.00191

Wang, X., Kim, Y., Hong, S.H., Ma, Q., Brown, B.L., Pu, M., Tarone, A.M., Benedik, M.J., Peti, W., Page, R., Wood, T.K., 2011. Antitoxin MqsA helps mediate the bacterial general stress response. Nat. Chem. Biol. 7, 359-366. doi:10.1038/nchembio.560

Yamaguchi, Y., Park, J.-H., Inouye, M., 2011. Toxin-antitoxin systems in bacteria and archaea. Annu. Rev. Genet. 45, 61-79. doi:10.1146/annurev-genet-110410-132412 
A

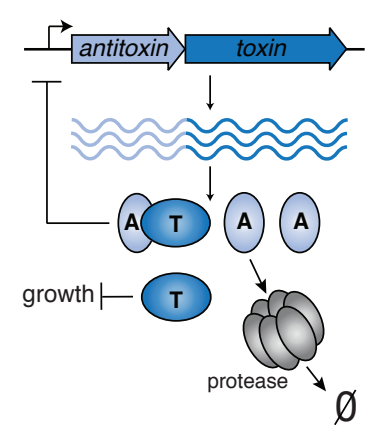

B

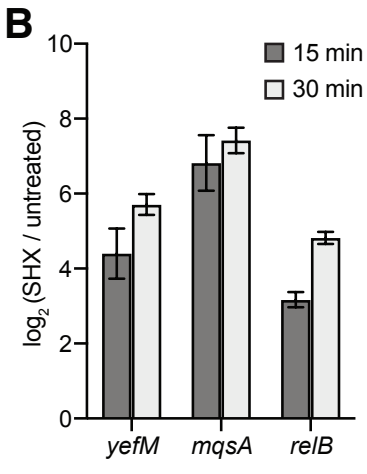

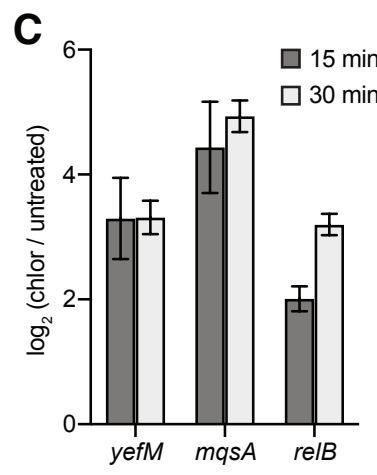

D

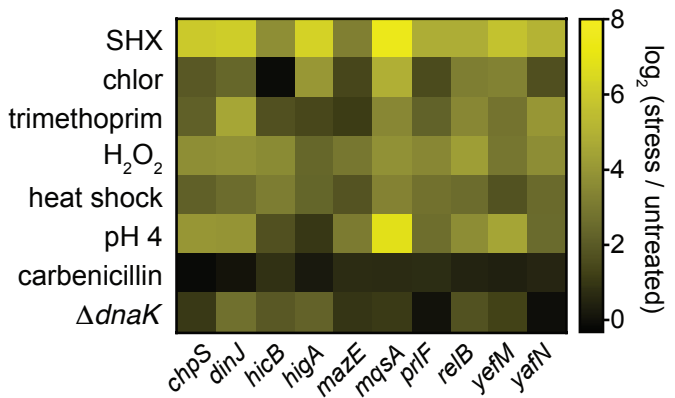

Figure 1. TA systems are transcriptionally induced by diverse stresses.

(A) Schematic summarizing key properties of toxin-antitoxin systems. Toxin and antitoxin are co-transcribed from a single operon, but the antitoxin is translated at a higher rate. Antitoxins bind to and repress their own promoters. Antitoxins are also substrates of cellular proteases such as Lon.

(B-C) Changes in antitoxin transcript level in response to serine hydroxamate (SHX) (B) or chloramphenicol (chlor) (C) as measured by qRT-PCR at the indicated time points. Antitoxin levels were normalized to the housekeeping gene gyrA and each stress normalized to measurements made for untreated cells.

(D) Difference in antitoxin transcript levels between cells exposed to stress for 30 minutes compared to untreated cells as measured by qRT-PCR.

Data are the average of 3 biological replicates with error bars indicating the S.D.

Also see Figure S1. 
bioRxiv preprint doi: https://doi.org/10.1101/2020.03.02.972737; this version posted March 3, 2020. The copyright holder for this preprint (which was not certified by peer review) is the author/funder, who has granted bioRxiv a license to display the preprint in perpetuity. It is made available under aCC-BY-NC-ND 4.0 International license.

A

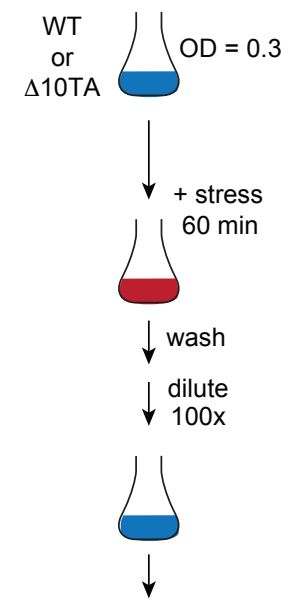

growth curve

$\mathbf{J}$

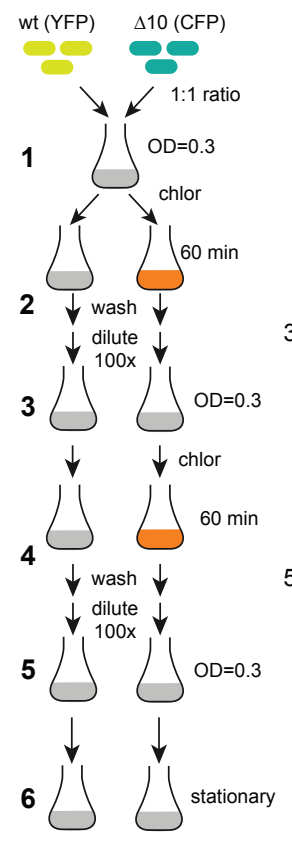

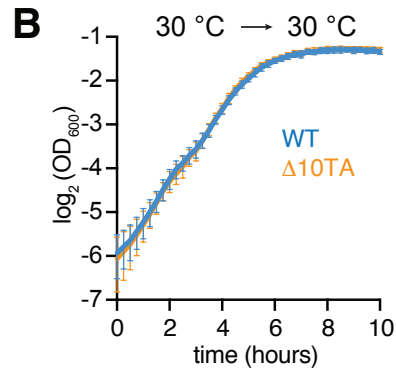
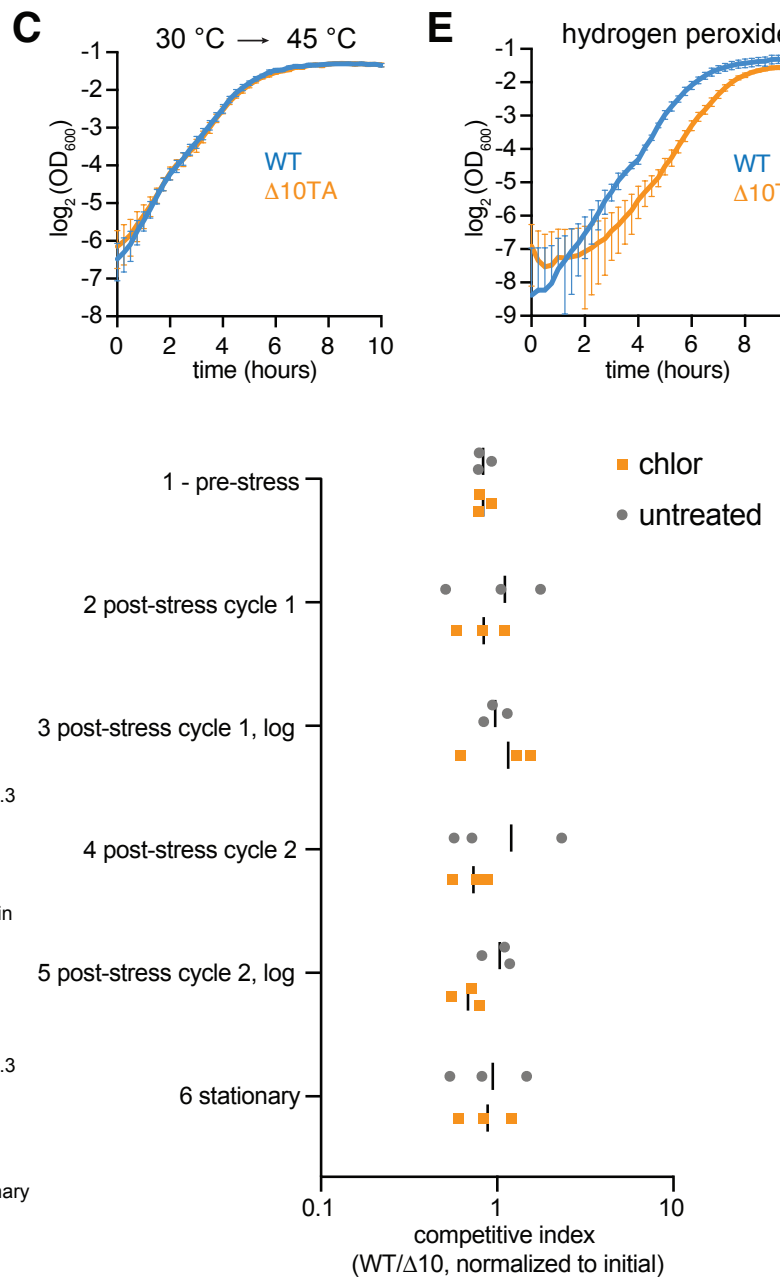

E
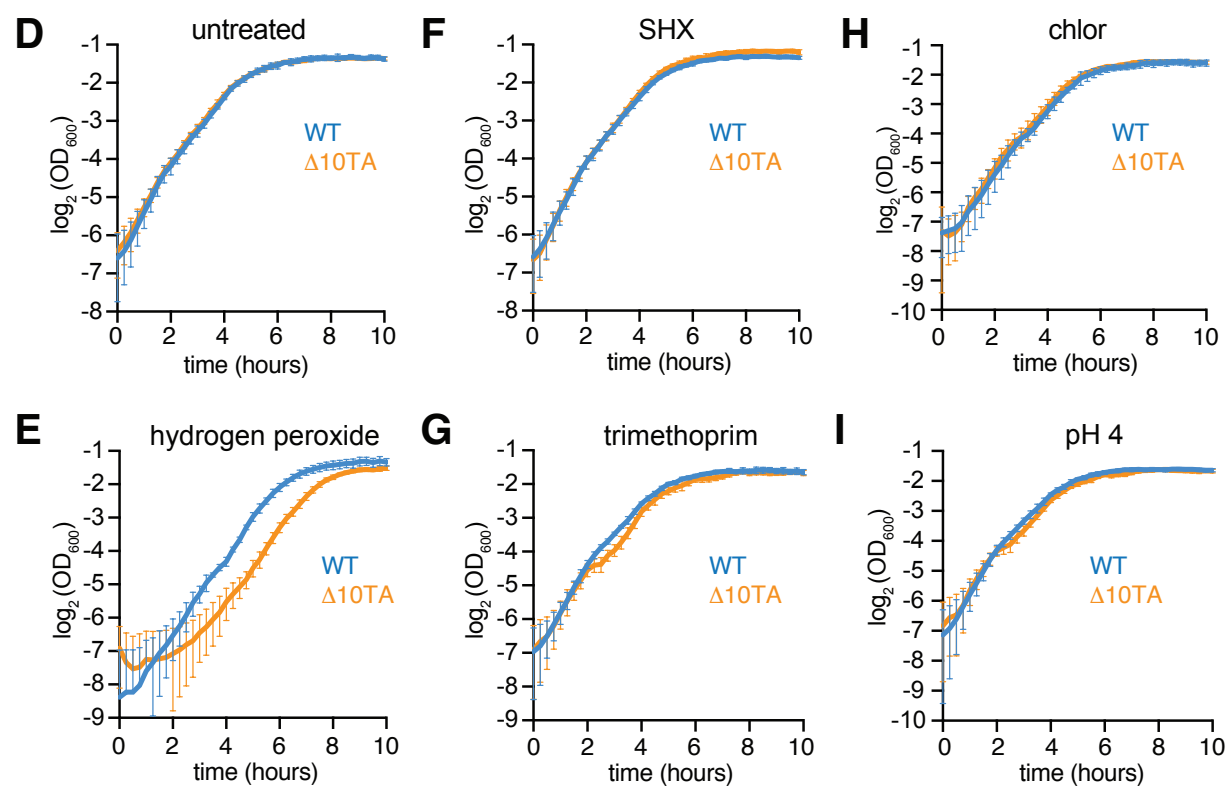

K

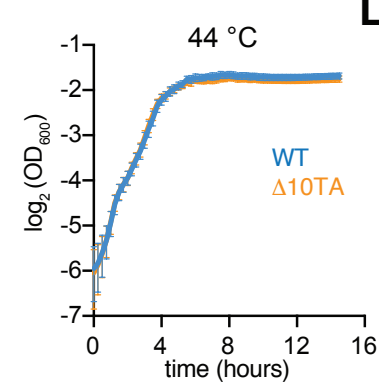

M
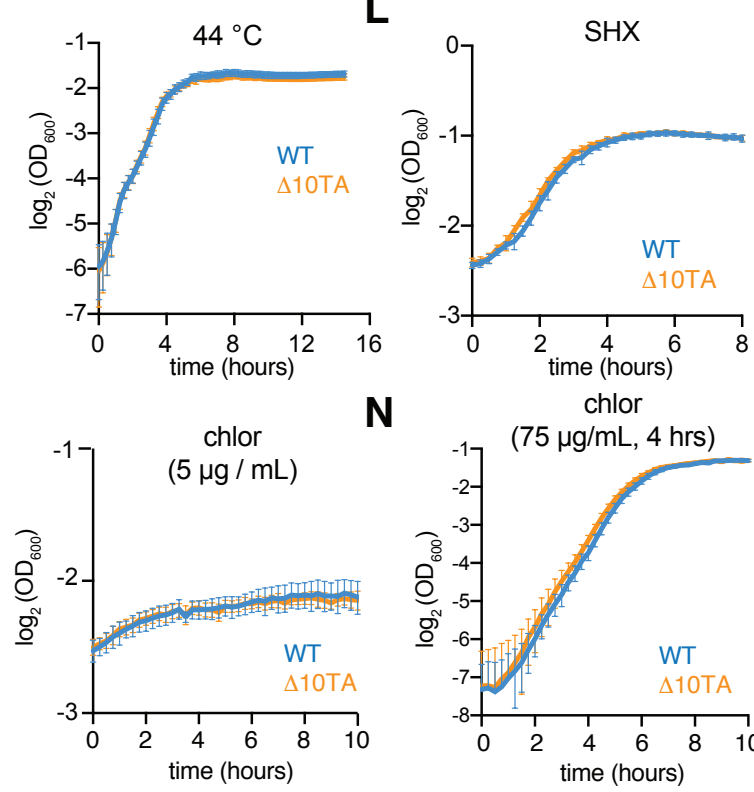

$\mathbf{N}$

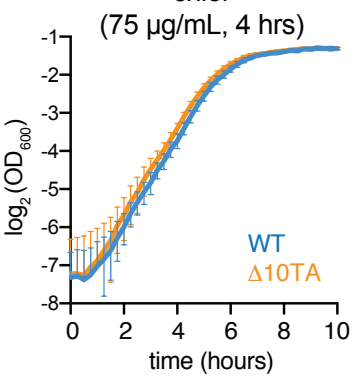

Figure 2. TA systems do not inhibit growth following diverse stresses.

(A) Experimental design for panels A-J to measure the growth of wild-type and $\triangle 10 T A$ strains following stress exposure.

(B-C) Growth curves for WT and $\triangle 10 \mathrm{TA}$ strains grown at $30{ }^{\circ} \mathrm{C}$ to $\mathrm{OD}_{600}$ of $\sim 0.3$, then maintained at $30^{\circ} \mathrm{C}$ (B) or shifted to $45^{\circ} \mathrm{C}(\mathrm{C})$ for 60 min before dilution and growth rate measurement at $37^{\circ} \mathrm{C}$ in flasks.

(D-I) Growth curves for WT and $\triangle 10 T A$ strains grown at $37^{\circ} \mathrm{C}$ and left untreated (D) or treated with the indicated stress for one hour then washed and diluted into fresh media, as described in (A). For complete description of stress conditions, see Methods.

(J) Wild-type and $\triangle 10 T A$ strains labeled with YFP and CFP, respectively, were mixed at a 1:1 ratio. This mixture was propagated and treated with two cycles of chloramphenicol as depicted in the schematic. Samples were taken points indicated by numbers on left, and colony forming units (c.f.u.) of the two strains were determined. The resulting competitive index is graphed on the right where individual symbols indicate independent replicates; black bar represents mean.

(K) Growth curve of WT and $\triangle 10 T A$ strains grown at $44^{\circ} \mathrm{C}$.

(L-M) Growth curve of WT and $\triangle 10 T A$ strains grown to $\mathrm{OD}_{600} \sim 0.3$, then treated with the indicated stress throughout growth measurements.

(N) Growth curves for WT and $\triangle 10 T A$ strains grown at $37^{\circ} \mathrm{C}$ and treated with chloramphenicol for four hours, then washed and diluted into fresh media, as described in (A). 
bioRxiv preprint doi: https://doi.org/10.1101/2020.03.02.972737; this version posted March 3, 2020. The copyright holder for this preprint (which was not certified by peer review) is the author/funder, who has granted bioRxiv a license to display the preprint in perpetuity. It is made available under aCC-BY-NC-ND 4.0 International license.

A
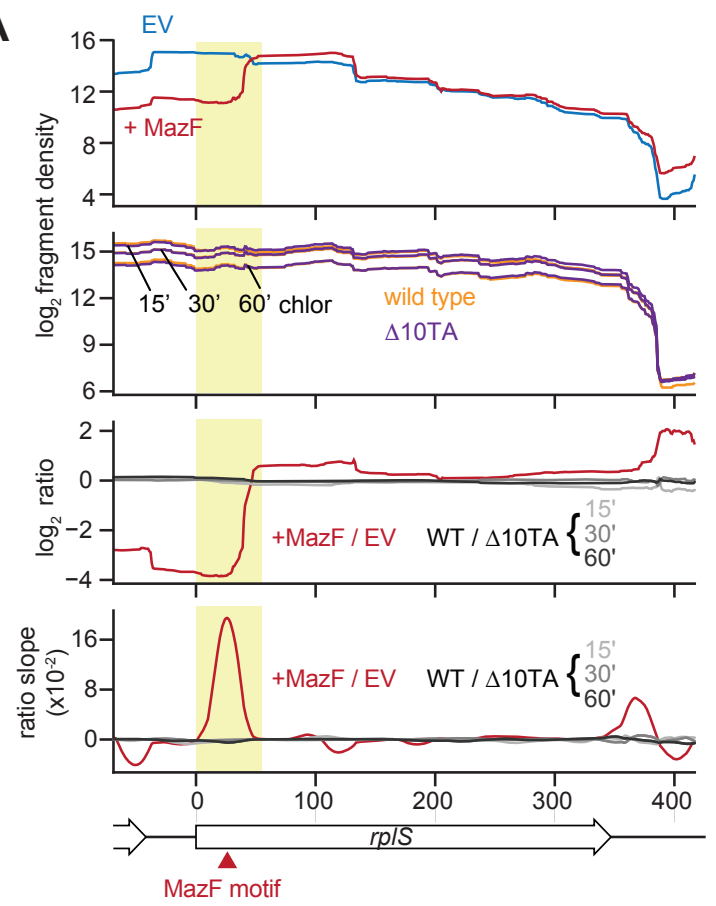

D

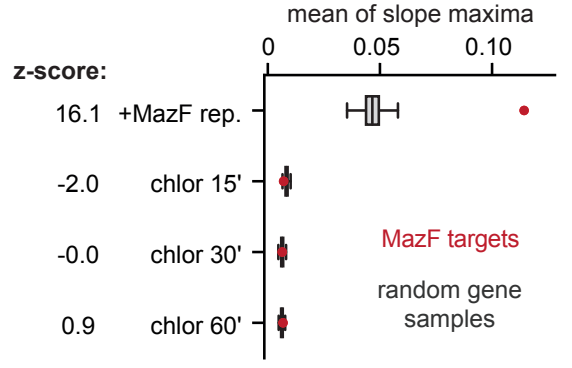

B
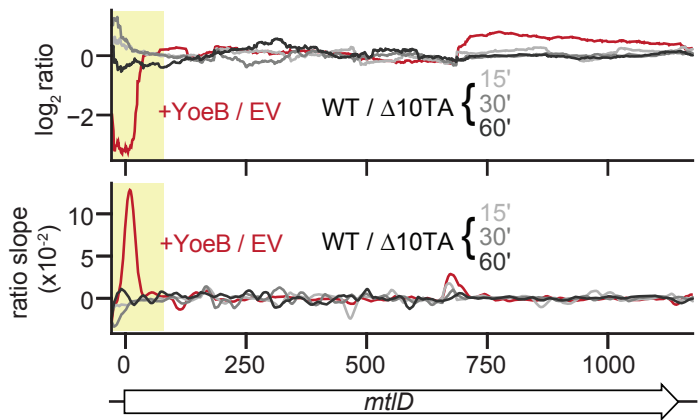

C
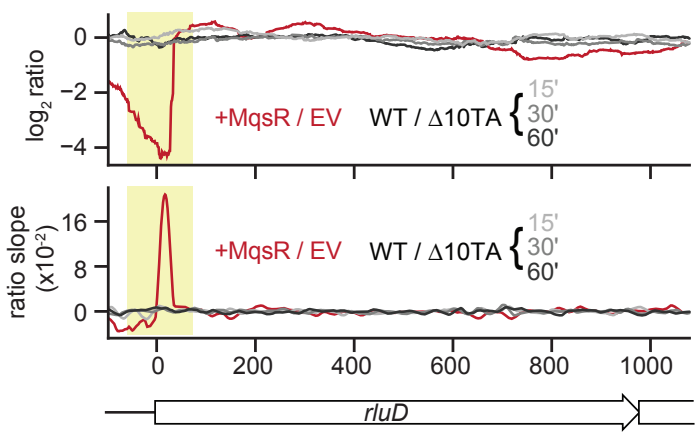

E

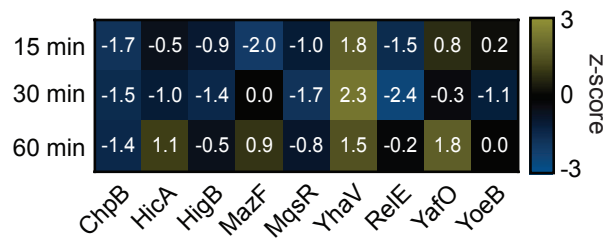

Figure 3. RNA-sequencing reveals no evidence of toxin activity

(A) Example analysis for MazF cleavage site (yellow shaded box) found within the rp/S transcript. Summed read counts for empty vector and MazF overexpression (upper panel) and wild-type or $\triangle 10 T A$ following chloramphenicol treatments at indicated time points (second panel). The ratios of MazF / empty vector and WT / $\triangle 10 T A$ in chloramphenicol are shown (third panel) with the slope of the ratio profiles below (fourth panel). The known MazF cleavage motif is indicated (red arrow).

(B-C) Ratio profiles (upper) and resulting ratio slopes (lower) are shown for a YoeB cleavage site (yellow box) in the $m t I D$ transcript (B) and MqsR cleavage site (yellow box) in the rluD transcript (C).

(D) Average of the slope maxima in the ratio profiles of coding regions corresponding to 'MazF targets' as defined in the text (red dot), compared to a distribution generated from sampling random sets of coding regions of the same size 10,000 times from the same sample (grey box and whiskers). The z-score calculated from this comparison is indicated (left). The same analysis was performed on the WT v. $\Delta 10 T A$ in chloramphenicol data sets, comparing MazF targets to a distribution based on random samples.

(E) Z-scores resulting from the analysis described in (D), but for each of the nine TA systems indicated.

See also Figures S2 and S3. 
bioRxiv preprint doi: https://doi.org/10.1101/2020.03.02.972737; this version posted March 3, 2020. The copyright holder for this preprint (which was not certified by peer review) is the author/funder, who has granted bioRxiv a license to display the preprint in perpetuity. It is made available under aCC-BY-NC-ND 4.0 International license.

A

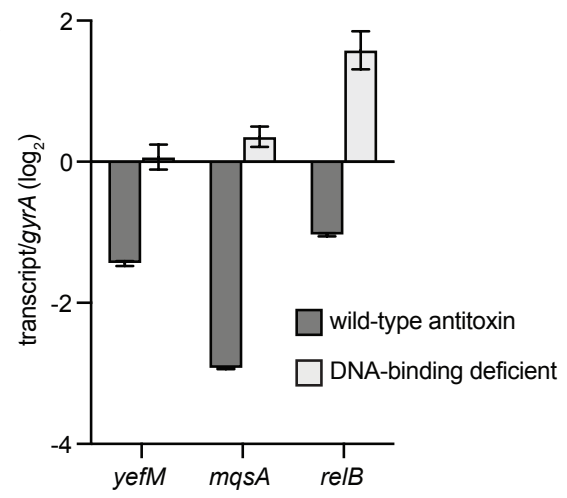

B

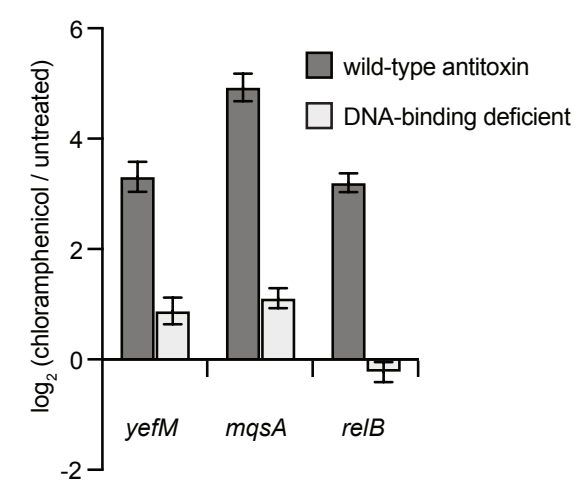

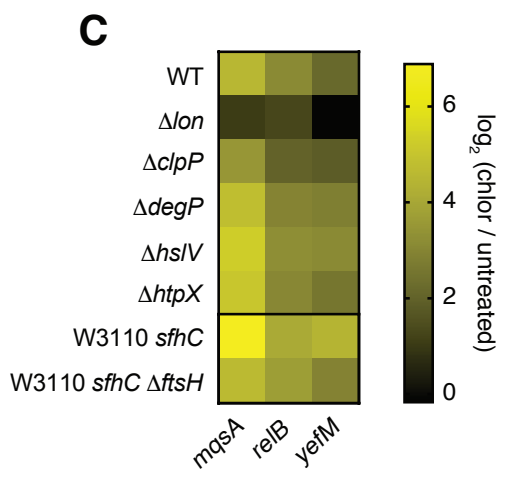

Figure 4. Increases in TA transcription result from a relief of autorepression.

(A) Antitoxin transcript levels were measured in untreated cells at $\mathrm{OD}_{600} \sim 0.3$ by qRT-PCR for the wild-type and corresponding DNA-binding mutant allele for the indicated antitoxin. Transcripts were normalized to the gyrA housekeeping gene. Data are the average of 3 biological replicates with error bars indicating the S.D.

(B) Changes in antitoxin transcript levels in chloramphenicol-treated compared to untreated cells as measured by qRT-PCR. Mutations abrogating DNA-binding by each antitoxin were introduced on the chromosome. Data are the average of 3 biological replicates with error bars indicating the S.D.

(C) Changes in antitoxin levels for chloramphenicol-treated cells compared with untreated cells of the wild-type strain or the indicated protease deletion mutant. Transcript levels were quantified by qRT-PCR. Data are the average of 2 biological replicates with error bars representing the S.D. 
bioRxiv preprint doi: https://doi.org/10.1101/2020.03.02.972737; this version posted March 3, 2020. The copyright holder for this preprint (which was not certified by peer review) is the author/funder, who has granted bioRxiv a license to display the preprint in perpetuity. It is made available under aCC-BY-NC-ND 4.0 International license.

A
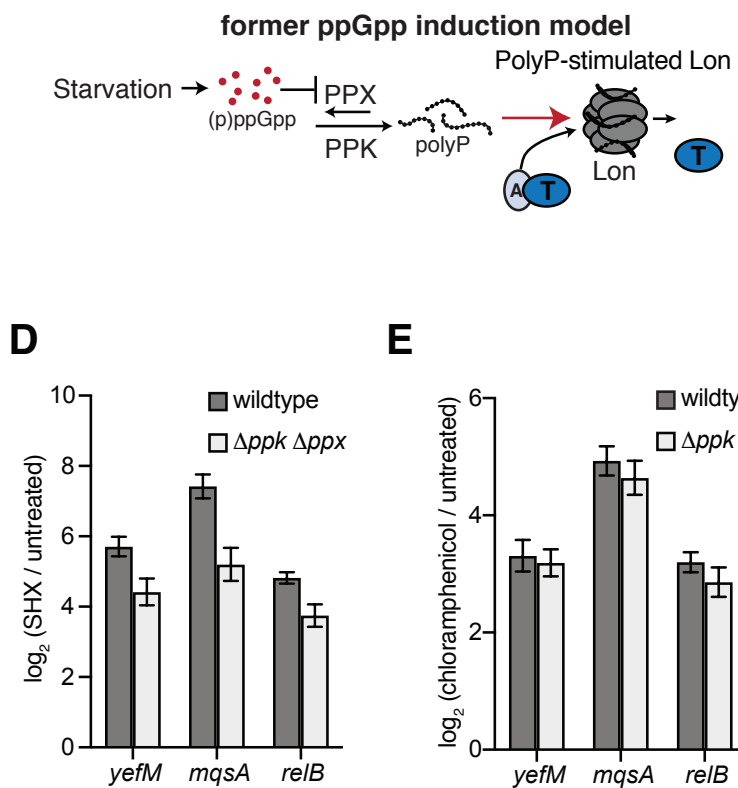

E

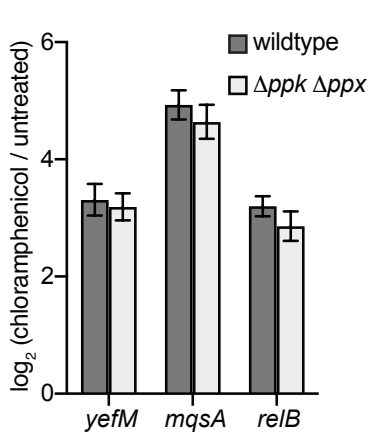

B

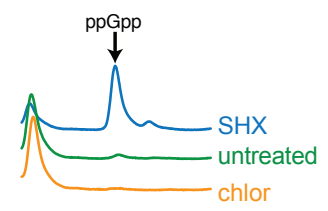

$\mathbf{F}$
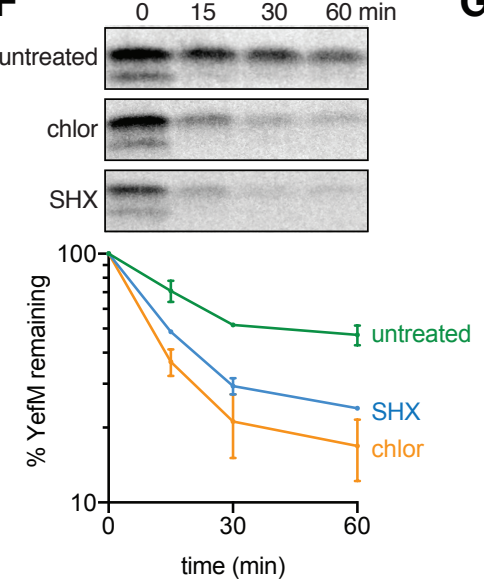

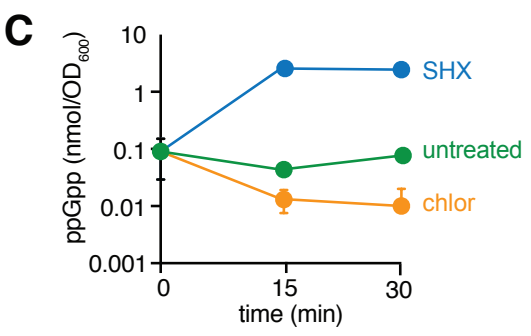

G

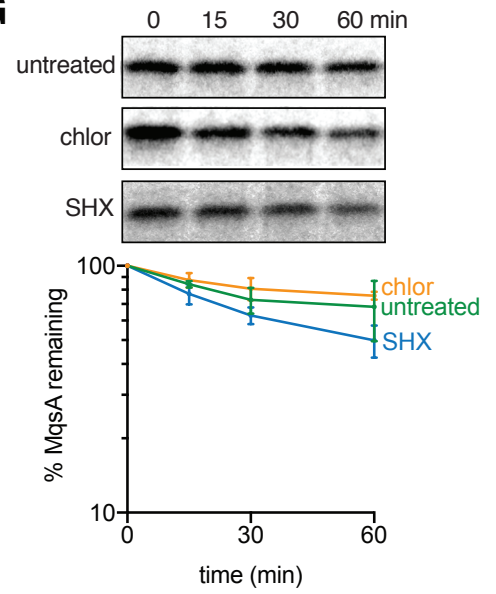

Figure 5. ppGpp is not required for TA transcription.

(A) Previous model for ppGpp-mediated activation of TA systems proposed by Maisonneuve et. al. (2013). Amino-acid starvation induces ppGpp, which inhibits polyphosphatase (Ppx), allowing polyphosphate kinase (Ppk) to synthesize polyphosphate, which then stimulates the Lon protease to degrade antitoxins and liberate toxins.

(B) Anion exchange chromatography traces for nucleotide extracts from cells harvested 30 min after the indicated treatment. Arrow indicates peak corresponding to ppGpp.

(C) Quantification of ppGpp levels in cells after the indicated treatments. Data points are the average of 3 biological replicates with error bars indicating S.D.

(D-E) Change in antitoxin transcript levels in cells treated with either SHX (D) or chloramphenicol (E) for 30 minutes compared to untreated cells. Transcript levels were quantified by qRT-PCR. Errors bars represent S.D. and data are the average of 3 biological replicates.

(F-G) Antitoxin degradation as measured by pulse-chase analysis. Strains bearing a medium-copy plasmid harboring a TA system under the control of its native promoter, pBR322-mqsRA (F) or pBR322-his6-yefM-yoeB (G), were grown to $\mathrm{OD}_{600} \sim 0.3$, then pulsed for 10 min with radiolabeled ${ }^{35} \mathrm{~S}$ cysteine and methionine, and chloramphenicol or $\mathrm{SHX}$ added at the same time as the unlabeled "chase" cysteine and methionine. Cell pellets were subsequently lysed and antitoxin was immunoprecipitated and analyzed by autoradiography. Representative gels are shown above with a quantification of 2 biological replicates shown below. 
bioRxiv preprint doi: https://doi.org/10.1101/2020.03.02.972737; this version posted March 3, 2020. The copyright holder for this preprint (which was not certified by peer review) is the author/funder, who has granted bioRxiv a license to display the preprint in perpetuity. It is made

A

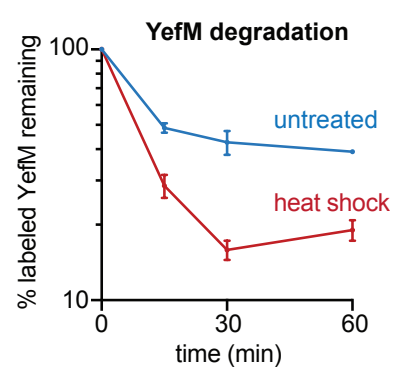

D

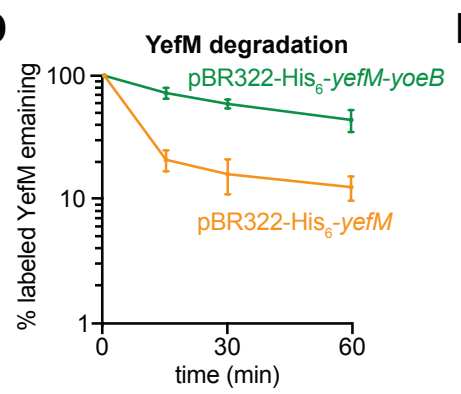

H

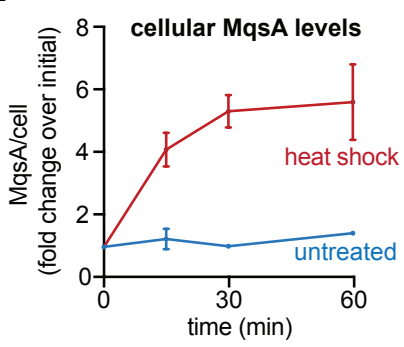

B

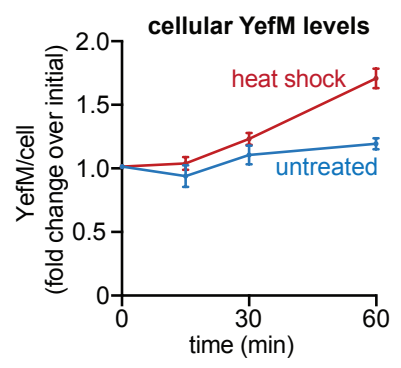

E

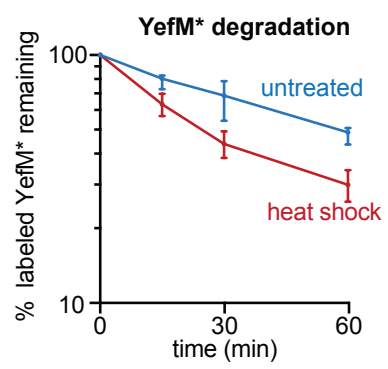

I

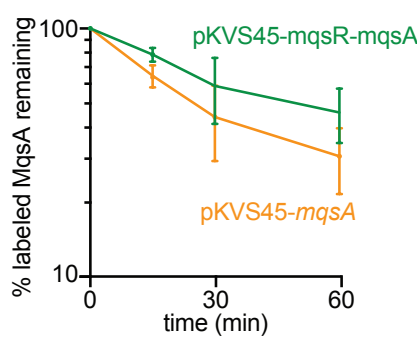

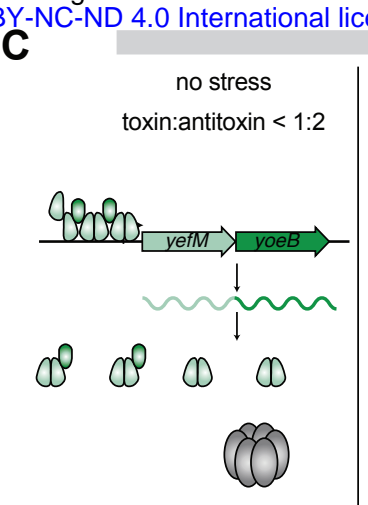

$\mathbf{F}$
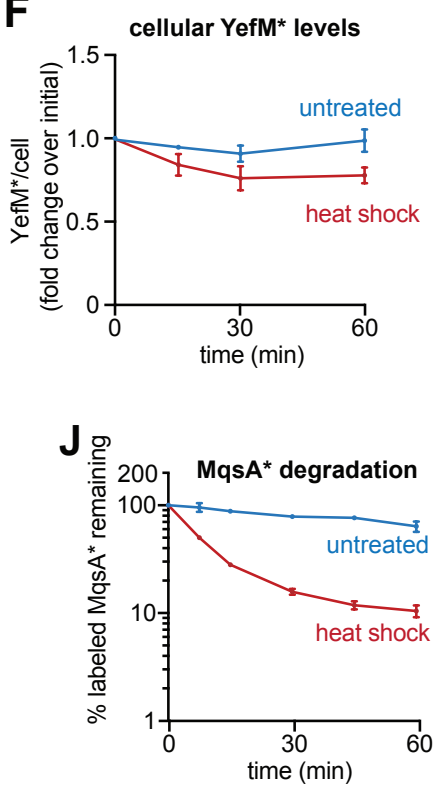

+ stress

toxin:antitoxin $\sim 1: 1$
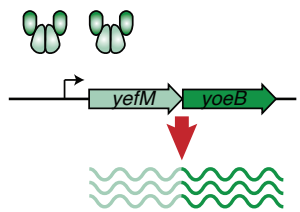

transcriptional induction

Figure 6. Autoregulation maintains antitoxin homeostasis despite changes in degradation.

(A) Antitoxin degradation rate of $\mathrm{His}_{6}-$ YefM (A) measured by pulse-chase analysis in cells grown at $30{ }^{\circ} \mathrm{C}$ and then either kept at 30 ${ }^{\circ} \mathrm{C}$ (untreated) or shifted to $45^{\circ} \mathrm{C}$ (heat shock).

(B) Cellular antitoxin levels were measured for $\mathrm{His}_{6}$-YefM by steady-state radiolabeling followed by immunoprecipitation and autoradiography using the same growth conditions as in panels $A$.

(C) Model for transcriptional induction of yefM-yoeB following a stress like heat shock. Stress leads to increased degradation of YefM antitoxin, particularly free antitoxin. As the ratio of toxin:antitoxin increases, YefM antitoxin cannot efficiently bind the yefM-yoeB promoter, leading to sustained transcriptional induction.

(D) Degradation rates measured by pulse-chase analysis for $\mathrm{His}_{6}$-YefM expressed with its cognate toxin, YoeB (green) or by itself (orange).

(E) Antitoxin degradation rate of $\mathrm{His}_{6}-\mathrm{YefM}^{*}$ measured as described in panel A.

(F) Cellular antitoxin levels of $\mathrm{His}_{6}-\mathrm{YefM}^{*}$ measured as described in panel B.

(G) Antitoxin degradation rate of MqsA measured as described in panel A.

(H) Cellular antitoxin levels of MqsA measured from wild-type cells by western blot.

(I) Degradation rates measured by pulse-chase analysis for MqsA expressed with its cognate toxin, MqsR (green) or by itself (orange).

(J) Antitoxin degradation rate of $\mathrm{Mqs}^{*}$ measured as described in panel A.

(K) Cellular antitoxin levels of MqsA* measured as described in panel $\mathrm{H}$. 
bioRxiv preprint doi: https://doi.org/10.1101/2020.03.02.972737; this version posted March 3, 2020. The copyright holder for this preprint (which was not certified by peer review) is the author/funder, who has granted bioRxiv a license to display the preprint in perpetuity. It is made available under aCC-BY-NC-ND 4.0 International license.
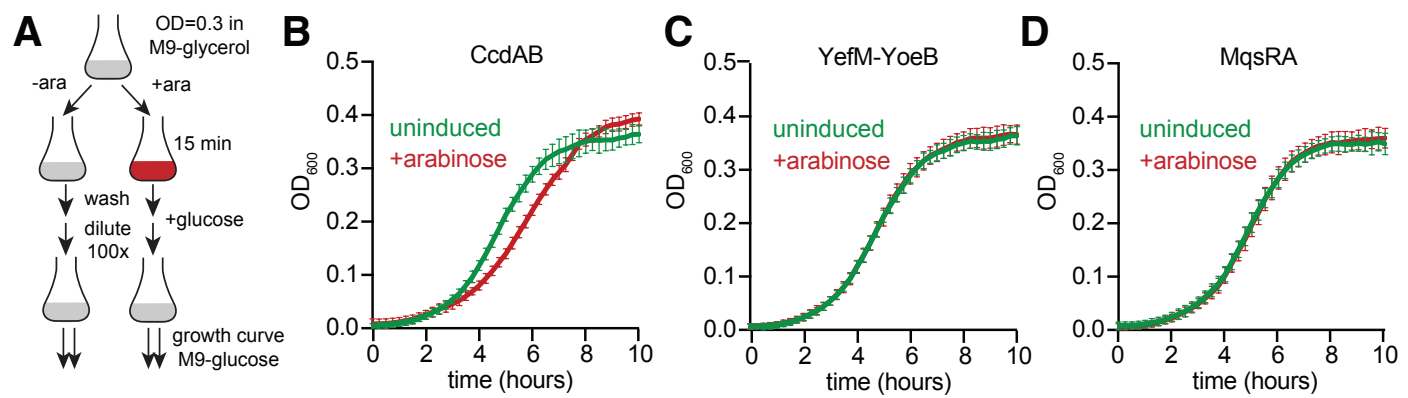

Figure 7. Chromosomal TA systems are not induced by an inhibition of new synthesis as with the plasmid-borne system CcdAB.

(A) Schematic for growth experiments in panels B-D. Strains were grown to $\mathrm{OD}_{600} \sim 0.3$ in flasks, then split into 2 flasks, one of which was induced with $0.2 \%$ arabinose for $15 \mathrm{~min}$. After washing, cells were released into repressing conditions (M9 media containing glucose) at a 100x dilution and growth was measured on a plate reader.

(B-D) Growth curves resulting from experiment described in panel A for (B) attL:: $\mathrm{P}_{\text {ara }}-c c d A B,(C) \triangle y e f M-y o e B$ attL:: $\mathrm{P}_{\text {ara }}-$ yefM-yoeB, and (D) $\triangle m q s R A$ attL:: $\mathrm{P}_{\text {ara }}-m q s R A$. 
bioRxiv preprint doi: https://doi org/10.1101/2020.03.02 972737; this version posted March 3, 2020. The copyright holder for this preprint (which was not certified by peer review) is the author/funder, who has granted bioRxiv a license to display the preprint in perpetuity. It is made available under aCC-BY-NC-ND 4.0 International license.

A

\section{SHX}

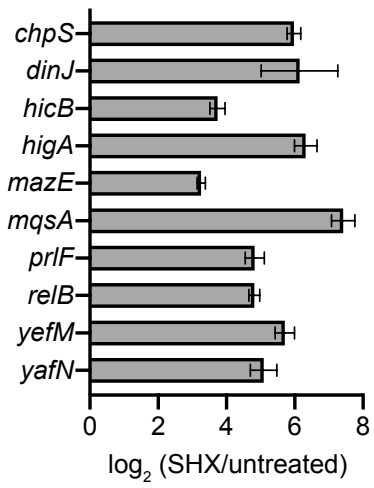

E

heat shock

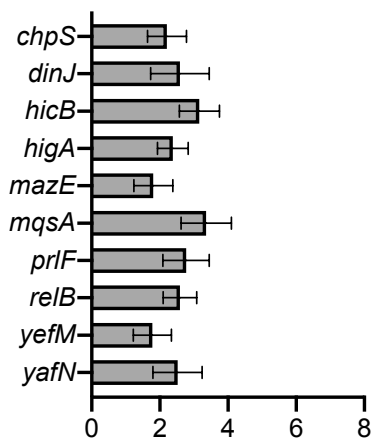

$\log _{2}$ (heat shock/untreated)
B

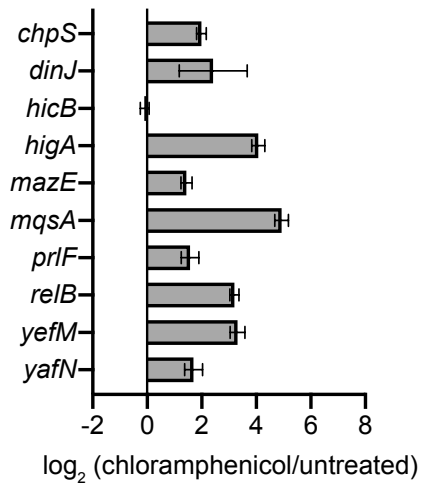

$\mathbf{F}$

$\mathrm{pH} 4$

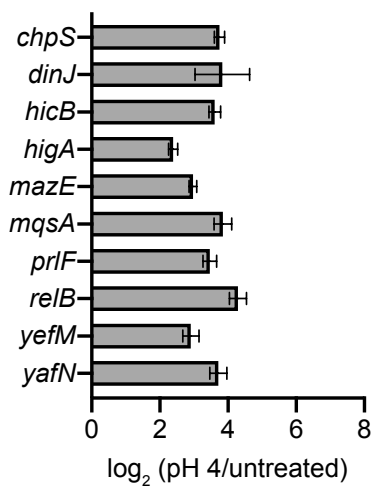

C trimethoprim

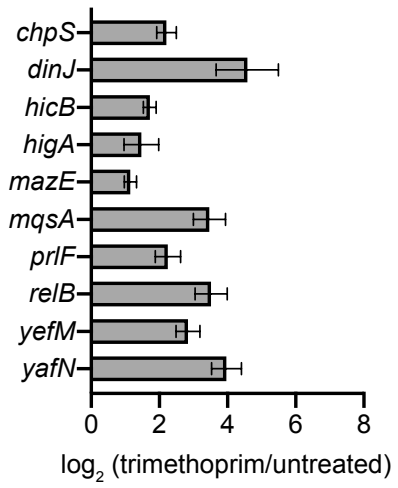

G

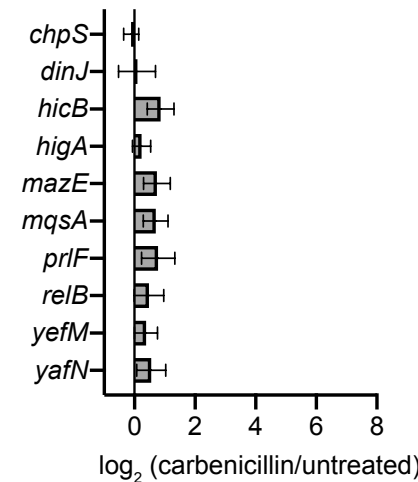

D hydrogen peroxide

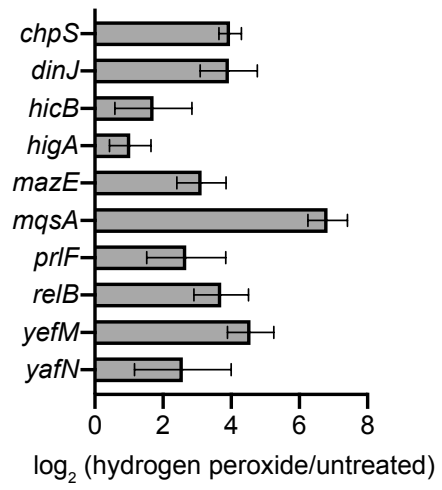

H

$\Delta d n a K$

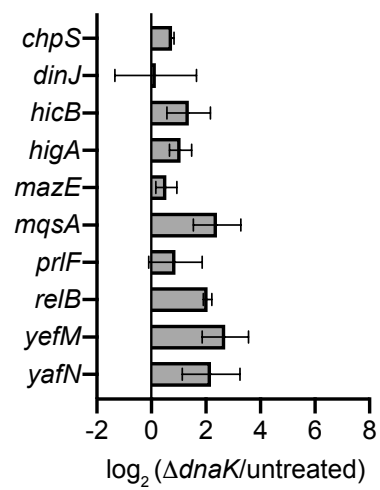

Figure S1. TA systems are transcriptionally activated by diverse stresses.

Difference in antitoxin transcript levels between cells exposed to the indicated stress for 30 minutes compared to untreated cells as measured by qRT-PCR corresponding to Figure 1D.

Data presented are the average of at least 3 biological replicates and errors bars represent S.D.

See also Figure 1. 
bioRxiv preprint doi: https://doi.org/10.1101/2020.03.02.972737; this version posted March 3, 2020. The copyright holder for this preprin (which was not certified by peer review) is the author/funder, who has granted bioRxiv a license to display the preprint in perpetuity. It is made

A
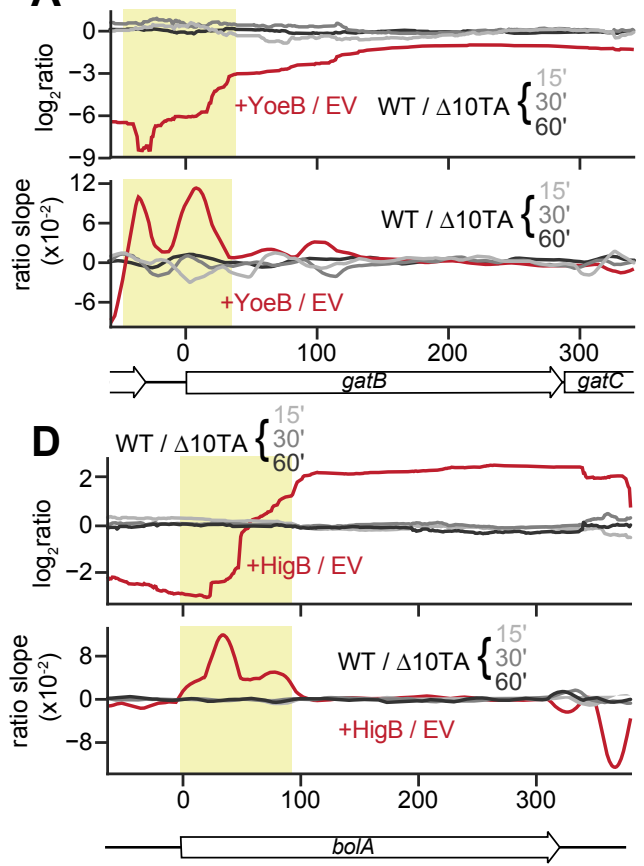

\section{G}
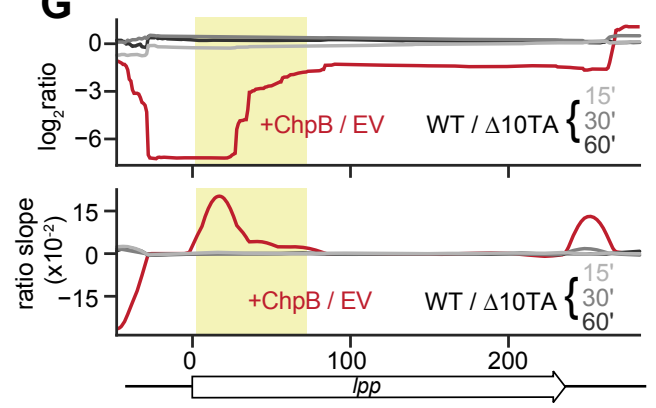

avail $\mathbf{B}$ le under aCC-BY-NC-ND 4.0 International license.C.
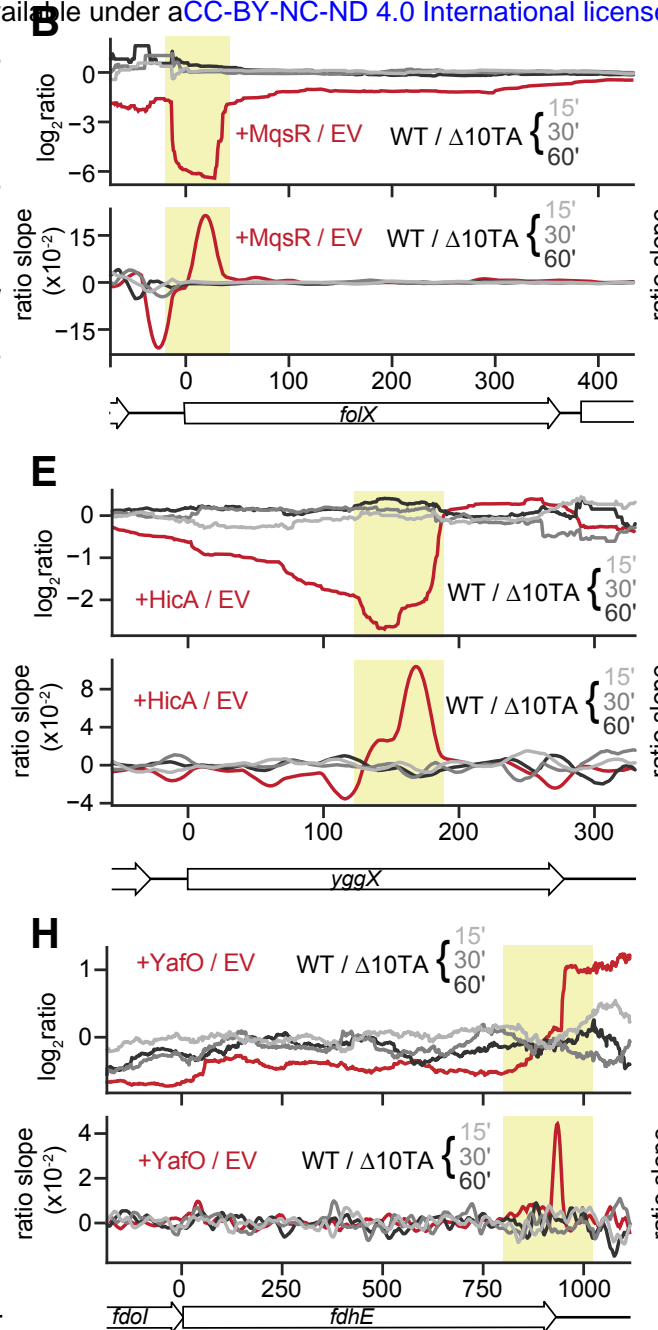
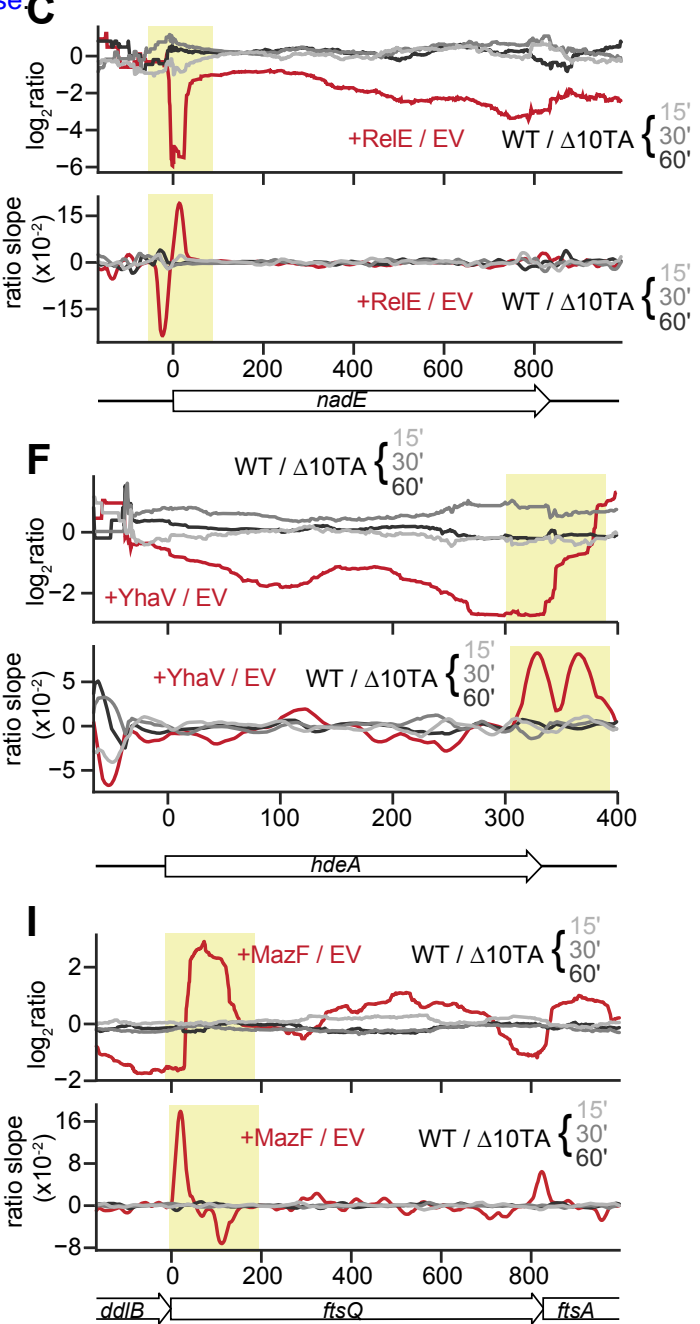

Figure S2. Transcripts targeted by toxins are not cleaved after chloramphenicol treatment.

(A-I) Examples of transcripts that show signatures of cleavage following toxin overexpression. Ratios of $+/$ - toxin and chloramphenicol-treated wild type / $\triangle 10 T A$ (upper panel) and corresponding slopes (lower panel) for each of the nine toxins examined. Toxin overexpression compared to empty vector is indicated in red, while wildtype/ $\Delta 10 T A$ following chloramphenicol treatment are in the indicated shade of gray.

See also Figure 3. 
bioRxiv preprint doi: https://doi.org/10.1101/2020.03.02.972737; this version posted March 3, 2020. The copyright holder for this preprint (which was not certified by peer review) is the author/funder, who has granted bioRxiv a license to display the preprint in perpetuity. It is made

A
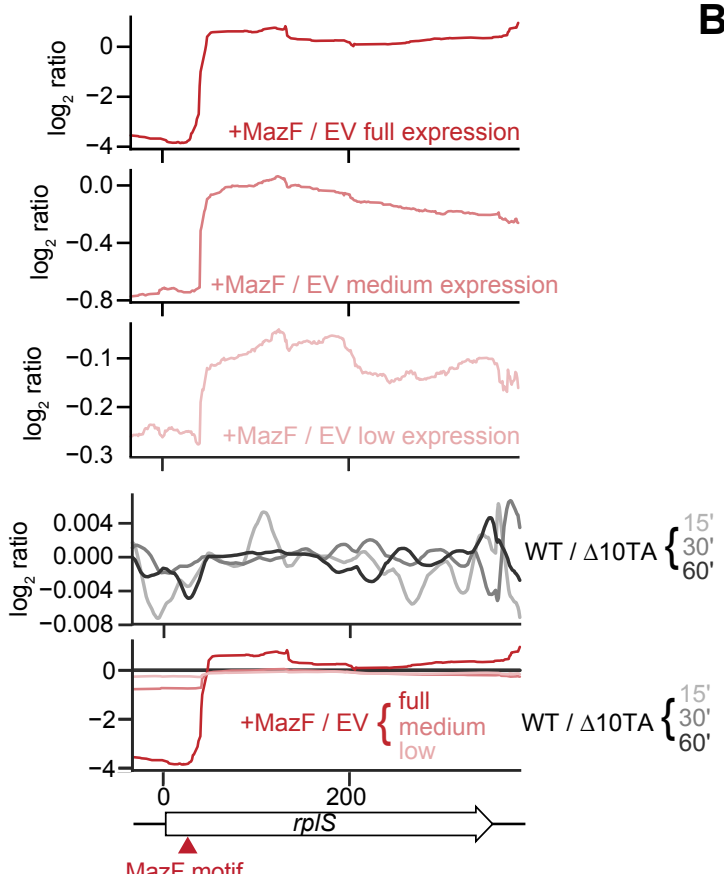

C

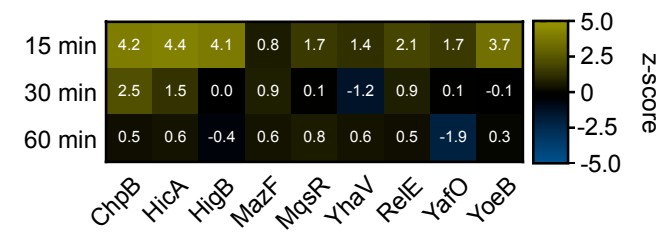

B
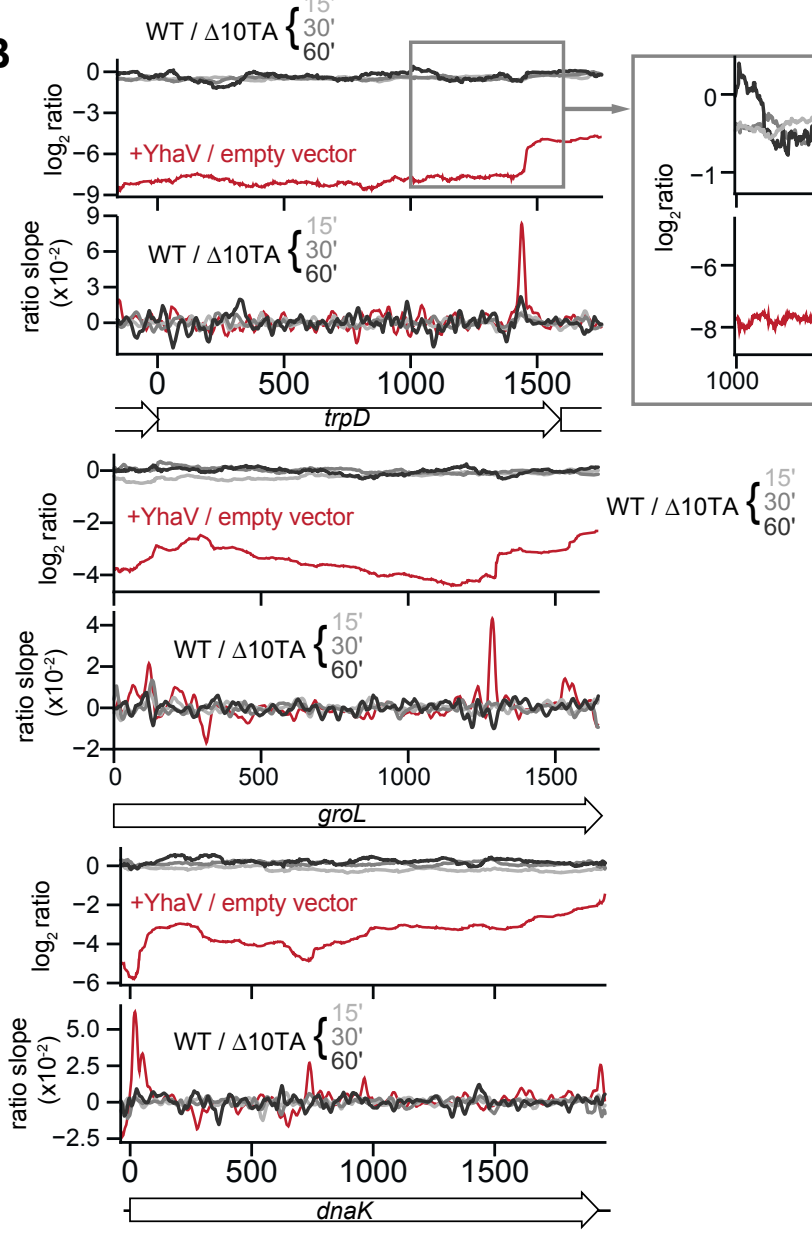

Figure S3. RNA-sequencing reveals no evidence of toxin activity

(A) Comparison of of the shape of ratio plots of + MazF / empty vector for rpiS transcript containing a MazF cleavage site at high, medium, and low MazF expression levels (upper 3 panels) and for wild type / $\Delta 10 T A$ in chloramphenicol treatment samples (fourth panel). Lower panel is an overlay of all five ratio profiles plotted on the same scale.

(B) YhaV regions containing the highest cleavage ratio slopes corresponding to analysis described in Fig. 3E. Chloramphenicol-treated samples are compared to YhaV overexpression data.

(C) Z-scores generated from comparing the average of the minima detected in the transcripts with the lowest $+/$ - toxin ratios (lowest $5 \%$, from toxin overexpression datasets) to the average of the minima from a randomly selected set of transcripts (sampled 10,000 times) in the chloramphenicol RNA-seq datasets. A negative z-score indicates a significant amount of toxin activity; positive z-scores may result from transcript stabilization.

See also Figure 3. 
bioRxiv preprint doi: https://doi.org/10.1101/2020.03.02.972737; this version posted March 3, 2020. The copyright holder for this preprint (which was not certified by peer review) is the author/funder, who has granted bioRxiv a license to display the preprint in perpetuity. It is made available under aCC-BY-NC-ND 4.0 International license.

A

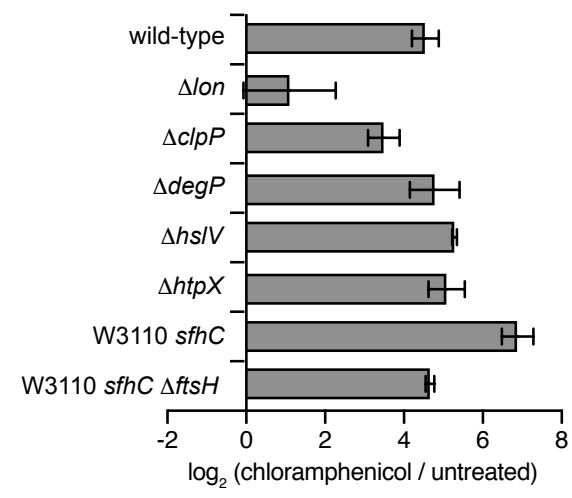

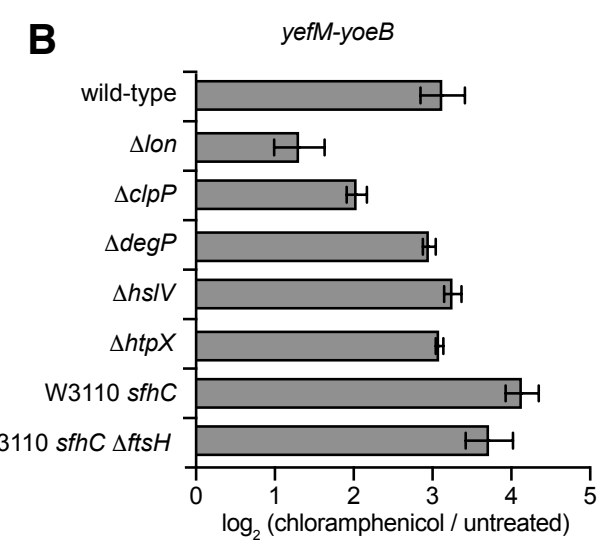

Figure S4. Chloramphenicol-induced changes in transcription require the Lon protease.

A-C) Difference in antitoxin levels of chloramphenicol-treated compared with untreated cells of the indicated protease deletion mutants for mqsRA (A), yefM-yoeB (B), and relBE (C). Transcript levels were quantified by qRT-PCR and correspond to Fig. 4B.

Data presented are the average of 2 biological replicates and error bars represent S.D.

See also Figure 4. 
bioRxiv preprint doi: https://doi.org/10.1101/2020.03.02 972737 ; this version posted March 3, 2020. The copyright holder for this preprint (which was not certified by peer review) is the author/funder, who has granted bioRxiv a license to display the preprint in perpetuity. It is made

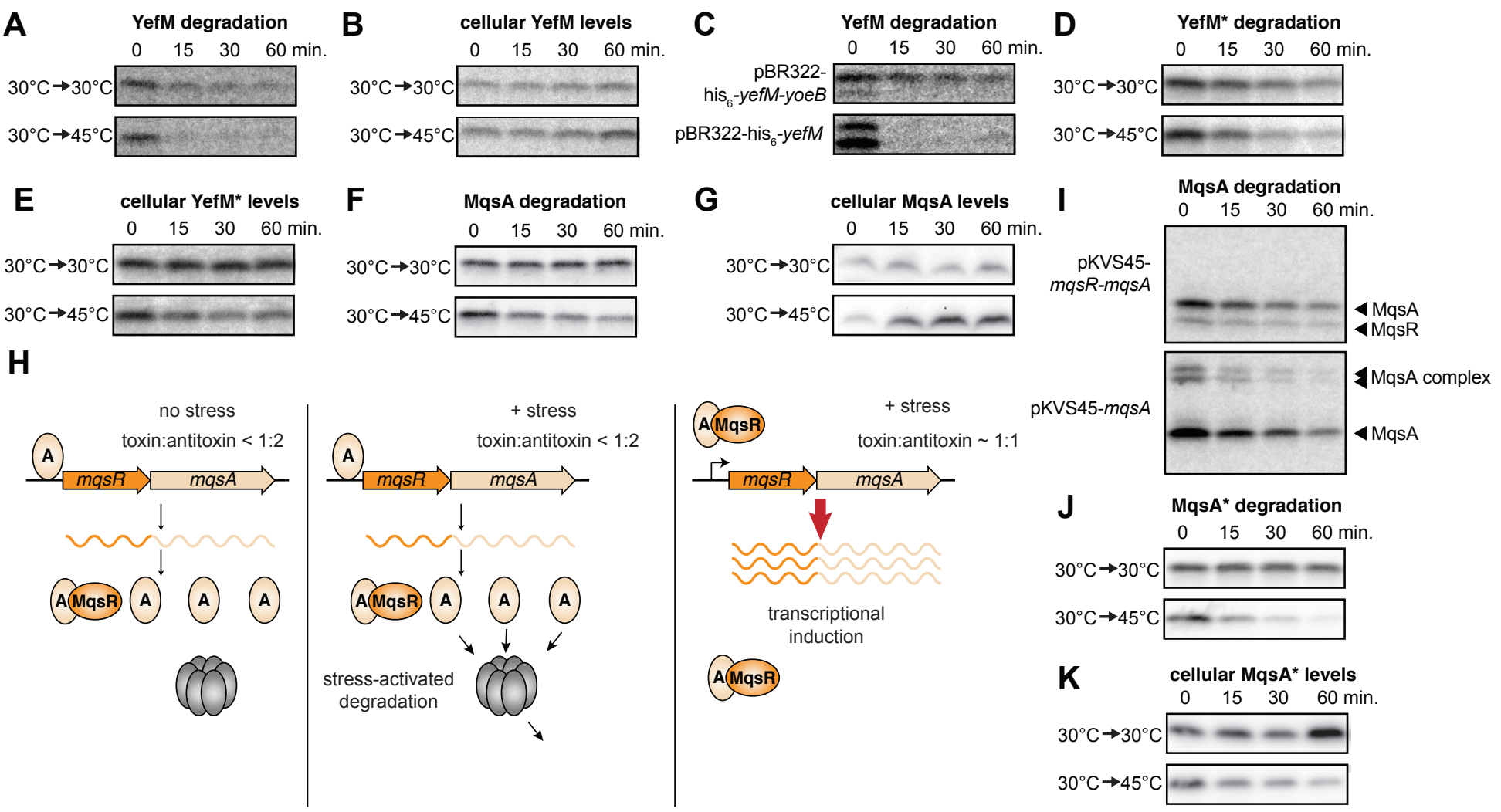

Figure S5. Autoregulation maintains antitoxin homeostasis despite changes in degradation.

(A-B) Representative phosphorimages of $\mathrm{His}_{6}-$ YefM degradation (A) or cellular antitoxin levels (B) in cells grown at $30{ }^{\circ} \mathrm{C}$ and then either kept at $30{ }^{\circ} \mathrm{C}$ (untreated) or shifted to $45^{\circ} \mathrm{C}$ (heat shock). Degradation was measured by pulse-chase and immunoprecipitation while cellular levels were measured by steady-state radiolabeling followed by immunoprecipitation and autoradiography.

(C) Representative phosphorimages of His6-YefM degradation following co-expression with its cognate toxin, YoeB (upper), or expression by itself (lower).

(D-E) Same as panels A-B but for His ${ }_{6}-$ YefM* $^{*}$.

(F-G) Representative phosphorimages of MqsA degradation measured by pulse chase ((F) or Western blots of cellular MqsA levels $(G)$ under growth conditions described in $(A)$.

(H) Model for MqsA protein levels following stress. MqsA cannot bind both DNA and MqsR, therefore at higher degradation rates as toxin:antitoxin levels increase, the promoter is derepressed, leading to transcriptional activation.

(I) Representative phosphorimages of MqsA degradation following co-expression with its cognate toxin, MqsR (upper), or expression by itself (lower).

(J-K) Same as panels F-G but for MqsA*.

See also Figure 6. 
bioRxiv preprint doi: https://doi.org/10.1101/2020.03.02.972737; this version posted March 3, 2020. The copyright holder for this preprint (which was not certified by peer review) is the author/funder, who has granted bioRxiv a license to display the preprint in perpetuity. It is made available under aCC-BY-NC-ND 4.0 International license.

Table S1. Primers used in this study

\begin{tabular}{|c|c|c|}
\hline Number & Purpose & Sequence (5'-3') \\
\hline 1 & $\begin{array}{l}\text { Linearize pBR322 without } \\
\text { tetracycline promoter }\end{array}$ & GGCACCTCGCTAACGGAT \\
\hline 2 & $\begin{array}{l}\text { Linearize pBR322 without } \\
\text { promoter }\end{array}$ & ACATGAGAATTCTTGAAGACGAAAGG \\
\hline 3 & yefM-yoeB into $\mathrm{pBR} 322$ & $\begin{array}{l}\text { GCTCCAATTCTTGGAGTGGTGAATCCGTTAGCGAGGTGCCTCTATA } \\
\text { AGGCTACGCTAGCG }\end{array}$ \\
\hline 4 & yefM-yoeB into $\mathrm{pBR} 322$ & $\begin{array}{l}\text { CGTATCACGAGGCCCTTTCGTCTTCAAGAATTCTCATGTTCACGTTA } \\
\text { GAAAGCAAGGGCT }\end{array}$ \\
\hline 5 & $m q s R A$ into $\mathrm{pBR} 322$ & $\begin{array}{l}\text { GCGTATCACGAGGCCCTTTCGTCTTCAAGAATTCTCATGTTGACTCC } \\
\text { AGCTTCCCTTATAAA }\end{array}$ \\
\hline 6 & $m q s R A$ into $\mathrm{pBR} 322$ & $\begin{array}{l}\text { GCTCCAATTCTTGGAGTGGTGAATCCGTTAGCGAGGTGCCAAGAAA } \\
\text { AAAGCGAGTTGCC }\end{array}$ \\
\hline 7 & relBE into $\mathrm{pBR} 322$ & $\begin{array}{l}\text { CTCCAATTCTTGGAGTGGTGAATCCGTTAGCGAGGTGCCTTCTCCT } \\
\text { GTCAACGCAAAGCA }\end{array}$ \\
\hline 8 & relBE into $\mathrm{pBR} 322$ & $\begin{array}{l}\text { CGTATCACGAGGCCCTTTCGTCTTCAAGAATTCTCATGTGGCCGGA } \\
\text { CTCTTTAAAAACGA }\end{array}$ \\
\hline 9 & pIB279 into yefM-yoeB locus & $\begin{array}{l}\text { TAATTAACGCTCATCATTGTACAATGAACTGTACAAAAGAGGAGAT } \\
\text { TGACCCTTTTATGATTTTCTATCAAAC }\end{array}$ \\
\hline 10 & pIB279 into yefM-yoeB locus & $\begin{array}{l}\text { GGCTACGCTAGCGTATCAAAACTGACAATTCATTCTATGAATGAAT } \\
\text { CTGTCTTGGTCGGTCATTTCGAAC }\end{array}$ \\
\hline 11 & pIB279 into mqsRA locus & $\begin{array}{l}\text { CTGTAATTAACCTTTTAGGTTATAACTAAAGTAACAGGGAGGCGGG } \\
\text { GGTTCCTTTTATGATTTTCTATCAAAC }\end{array}$ \\
\hline 12 & pIB279 into $m q s R A$ locus & $\begin{array}{l}\text { CTCACACTCCGGTAAAGAAAAAAGCGAGTTGCCCCCGCTTTTCCAT } \\
\text { TAATCTTGGTCGGTCATTTCGAAC }\end{array}$ \\
\hline 13 & pIB279 into relBE locus & $\begin{array}{l}\text { TTGTAGTGCGATACTTGTAATGACATTTGTAATTACAAGAGGTGTA } \\
\text { AGACCCATCACATATACCTGCCGT }\end{array}$ \\
\hline 14 & pIB279 into relBE locus & $\begin{array}{l}\text { CAAAGCAGAAGTGTCACCTTCGGTGCGAAACAGAGATGTCATGCTT } \\
\text { TGGTCACGCTATCTGTGCAAGGTC }\end{array}$ \\
\hline 15 & yefM(R10A A9R) F & GCACAGAATTTGTCGGCAACAATGATG \\
\hline 16 & yefM(R10A A9R) R & CGCTTCGCTGTAGCTAATTGTAC \\
\hline 17 & $m q s A(\mathrm{~N} 97 \mathrm{~A} \mathrm{R} 101 \mathrm{~A}) \mathrm{F}$ & GCAGCGTTTTCGGCATACGAAAAAGGCAATGCCCA \\
\hline 18 & $m q s A(\mathrm{~N} 97 \mathrm{~A} \mathrm{R} 101 \mathrm{~A}) \mathrm{R}$ & TACACCTCCCCCAAAAATTTCGC \\
\hline 19 & $\operatorname{relB}(\mathrm{S} 28 \mathrm{~L}) \mathrm{R}$ & AGGAGTTACACCCATTTTTTCAAGC \\
\hline 20 & $\operatorname{relB}(\mathrm{S} 28 \mathrm{~L}) \mathrm{F}$ & CTAGAAGCGCTTCGTCTCATGC \\
\hline 21 & ppk-ppx deletion $\mathrm{F}$ & $\begin{array}{l}\text { TGTAAATCGCAAGCTCCAGCAGTTTTTTTC CCCCTTTTCT } \\
\text { GGCATAGTTGGTGTAGGCTGGAGCTGCTTC }\end{array}$ \\
\hline 22 & ppk-ppx deletion $\mathrm{R}$ & $\begin{array}{l}\text { GAAAGTGCCTGAATAATGCGGGCCGACATTTCTCGTCGGCCCGCAA } \\
\text { AGTAATGGGAATTAGCCATGGTCC }\end{array}$ \\
\hline 23 & Add his 6 tag to $y e f M$ & CATCACCACCATCACCATCGTACAATTAGCTACAGCGAAGC \\
\hline 24 & Add his6 tag to $y e f M$ & CATGTCAATCTCCTCTTTTGTACAGG \\
\hline 25 & $\begin{array}{l}\text { YefM F; for inverse PCR of } \\
\text { pBR322- His6-yefM-yoeB } \\
\text { removing yoeB sequence }\end{array}$ & TCACTCAATGATGTCCTTTTCCGT \\
\hline
\end{tabular}


bioRxiv preprint doi: https://doi.org/10.1101/2020.03.02.972737; this version posted March 3, 2020. The copyright holder for this preprint (which was not certified by peer review) is the author/funder, who has granted bioRxiv a license to display the preprint in perpetuity. It is made available under aCC-BY-NC-ND 4.0 International license.

\begin{tabular}{|c|c|c|}
\hline 26 & $\begin{array}{l}\text { Amplify YefM R; for inverse } \\
\text { PCR of pBR322-His6-yefM-yoeB } \\
\text { removing yoeB sequence }\end{array}$ & ACAGATTCATTCATAGAATGAATTGTC \\
\hline 27 & Insert $m q s R A$ into pKVS45 & ATGCATAGAGCTCAAGTAACAGGGAGGCGGGGGTT \\
\hline 28 & Insert $m q s R A$ into $\mathrm{pKVS} 45$ & AGTCAAAGCTTTTAACGGATTTCATTCAATAG \\
\hline 31 & $\begin{array}{l}\text { Insert } m q s R A \text { into pKVS45 with } \\
m q s A \text { RBS }\end{array}$ & AGTACTAGAGCTCAAGGAGAAGTAATATGAAATGTCCGGT \\
\hline 32 & $\begin{array}{l}\text { yefM-yoeB chromosomal } \\
\text { replacement }\end{array}$ & $\begin{array}{l}\text { TAATTAACGCTCATCATTGTACAATGAACTGTACAAAAGAGGAGAT } \\
\text { TGACATGCGTACAATTAGCTACAGCGA }\end{array}$ \\
\hline 33 & $\begin{array}{l}\text { yefM-yoeB chromosomal } \\
\text { replacement }\end{array}$ & $\begin{array}{l}\text { GGCTACGCTAGCGTATCAAAACTGACAATTCATTCTATGAATGAAT } \\
\text { CTGTTCAATAATGATAACGACATGCTGCA }\end{array}$ \\
\hline 34 & $\begin{array}{l}m q s R A \text { chromosomal } \\
\text { replacement }\end{array}$ & $\begin{array}{l}\text { CTGTAATTAACCTTTTAGGTTATAACTAAAGTAACAGGGAGGCGGG } \\
\text { GGTTATGGAAAAACGCACACCACAT }\end{array}$ \\
\hline 35 & $\begin{array}{l}m q s R A \text { chromosomal } \\
\text { replacement }\end{array}$ & $\begin{array}{l}\text { CTCACACTCCGGTAAAGAAAAAAGCGAGTTGCCCCCGCTTTTCCAT } \\
\text { TAATTTAACGGATTTCATTCAATAGTTCTGGATGC }\end{array}$ \\
\hline 36 & yefM-yoe $B$ deletion oligo & $\begin{array}{l}\text { ACAATTCATTCTATGAATGAATCTGTTCAATAATGATAACAATTGT } \\
\text { ACGCATGTCAATCTCCTCTTTTGTACAGTTCATT }\end{array}$ \\
\hline 37 & $m q s R A$ deletion oligo & $\begin{array}{l}\text { AGAAAAAAGCGAGTTGCCCCCGCTTTTCCATTAATTAACGTTCCAT } \\
\text { AACCCCCGCCTCCCTGTTACTTTAGTTATAACCT }\end{array}$ \\
\hline 38 & Linearize pAH150 & CATGTTTGACAGCTTATCACTGATCA \\
\hline 39 & Linearize pAH150 & CGGTACCTGCAGGTATGGAGAAAC \\
\hline 40 & Insert $c c d A B$ into pAH150 & $\begin{array}{l}\text { TACTGTTTCTCCATACCTGCAGGTACCGGATGTCAAAAAGAGGTGT } \\
\text { GCTATG }\end{array}$ \\
\hline 41 & Insert $c c d A B$ into $\mathrm{pAH} 150$ & $\begin{array}{l}\text { CACTGATCAGTGATAAGCTGTCAAACATGATTATATTCCCCAGAAC } \\
\text { ATCAGGT }\end{array}$ \\
\hline 42 & Insert yefM-yoeB into pAH150 & $\begin{array}{l}\text { TACTGTTTCTCCATACCTGCAGGTACCGGTCTGTACAAAAGAGGAG } \\
\text { ATTGACATGC }\end{array}$ \\
\hline 43 & Insert yefM-yoeB into pAH150 & $\begin{array}{l}\text { CACTGATCAGTGATAAGCTGTCAAACATGATCAATAATGATAACGA } \\
\text { CATGCTGCAATG }\end{array}$ \\
\hline 44 & Insert $m q s R A$ into pAH150 & $\begin{array}{l}\text { TACTGTTTCTCCATACCTGCAGGTACCGGTAACAGGGAGGCGGGGG } \\
\text { TTA }\end{array}$ \\
\hline 45 & Insert $m q s R A$ into pAH150 & $\begin{array}{l}\text { CACTGATCAGTGATAAGCTGTCAAACATGATTAACGGATTTCATTC } \\
\text { AATAGTTCTGGATGCT }\end{array}$ \\
\hline \multicolumn{3}{|c|}{ qRT-PCR primers } \\
\hline 44 & gyrA F & CTGGAGAAGCACAGAATA \\
\hline 45 & gyrA R & GCGGGAAAGGTAAATCT \\
\hline 46 & chpS_F & GCAGGTATGGTCATTCCCAA \\
\hline 47 & chpS_R & GAGATGGGTGTCAGAATCAGTT \\
\hline 48 & dinJ_F & CGCCCGAATCGATGAAGAT \\
\hline 49 & dinJ_R & GCGACCTTTGTGAGGGTTAT \\
\hline 50 & hicB_F & CGCCTCTAAGGTATTGCTGTTA \\
\hline 51 & hicB_R & GGCGAGTAATCTCCTGTTTAGG \\
\hline 52 & higA_F & GGGTATTCAGAACGAGGAACAA \\
\hline 53 & higA_R & CACACACCAGATCCAGCAA \\
\hline 54 & mazE_F & CTTCCTTATCTTTCGGCTCTCC \\
\hline 55 & mazE_R & TGCGTAAAGAGCCCGTATTT \\
\hline 56 & mqsA F & CAACACGTAGTGCCCTGTTA \\
\hline 57 & mqsA R & CAGAGTAGGTGGTCATGCTTT \\
\hline 58 & prlF_F & TACGCGGACAAACAACTATCC \\
\hline
\end{tabular}


bioRxiv preprint doi: https://doi.org/10.1101/2020.03.02.972737; this version posted March 3, 2020. The copyright holder for this preprint (which was not certified by peer review) is the author/funder, who has granted bioRxiv a license to display the preprint in perpetuity. It is made available under aCC-BY-NC-ND 4.0 International license.

\begin{tabular}{|l|l|l|}
\hline 59 & prlF_R & CCACCAGGCAGAATTTCGTA \\
\hline 60 & relB_F & CATGCTCGAGTATATCGCTGAC \\
\hline 61 & relB_R & CGAAGCCGTTCTTTCACTATCT \\
\hline 62 & yefM F & TTGAGTCCATCAATCTCC \\
\hline 63 & yefM R & GAGGCTTGTGTTCTGAT \\
\hline 64 & yafN_F & TCTTAAGTGCCAGCGCATTC \\
\hline 65 & yafN_R & CCTCTAATCTTGCAGCACTTGG \\
\hline
\end{tabular}


bioRxiv preprint doi: https://doi.org/10.1101/2020.03.02.972737; this version posted March 3, 2020. The copyright holder for this preprint (which was not certified by peer review) is the author/funder, who has granted bioRxiv a license to display the preprint in perpetuity. It is made available under aCC-BY-NC-ND 4.0 International license.

Table S2. Strains used in this study.

\begin{tabular}{|c|c|c|}
\hline Name & Genotype & Reference \\
\hline ML6 & MG1655 & \\
\hline ML3203 & dnaK::kan & This study \\
\hline ML3204 & $m q S A^{\text {N97A RI01A }}$ & This study \\
\hline ML3205 & $y e f M^{R 10 A}$ & This study \\
\hline ML3206 & $\mathrm{relB}^{S 28 L}$ & This study \\
\hline ML3207 & $\Delta l o n$ & This study \\
\hline ML3208 & $\Delta c l p P$ & This study \\
\hline ML3209 & $\operatorname{deg} P: \because k a n$ & This study \\
\hline ML3210 & hslV::kan & This study \\
\hline ML3211 & htpX::kan & This study \\
\hline ML3212 & $s f h C 21$ zad220::Tn10 & (Ogura et al., 1999) \\
\hline ML3213 & sfhC21 zad220::Tn10 $\Delta f t s h H 3:: k a n$ & (Ogura et al., 1999) \\
\hline ML3214 & $\Delta p p k \Delta p p x$ & This study \\
\hline ML3215 & pBR322-his ${ }_{6}-y e f M-y o e B$ & This study \\
\hline ML3216 & DyefM-yoeB pBR322-his ${ }_{6}-$ yefM(R10A)-yoeB & This study \\
\hline ML3217 & pBR322-his ${ }_{6}-y e f M$ & This study \\
\hline ML3218 & pBR322-mqsRA & This study \\
\hline ML3219 & pKVS45-mqsRA & This study \\
\hline ML3220 & pKVS45-mqsA & This study \\
\hline ML3221 & MG1655 & (Goeders et al., 2013) \\
\hline ML3222 & $\begin{array}{l}\Delta m a z E F \Delta r e l B E \Delta c h p B \triangle d i n J-y a f Q \Delta y e f M-y o e B \\
\Delta y a f N O \Delta p r l F-y h a V \triangle h i c A B \Delta y g j M N \Delta m q s R A\end{array}$ & (Goeders et al., 2013) \\
\hline ML3223 & att $L: \because P_{\text {ara }}-c c d A B$ & This study \\
\hline ML3224 & $\Delta y e f M-y o e B$ att $L:: P_{\text {ara }}-y e f M-y o e B$ & This study \\
\hline ML3225 & $\Delta m q s R A$ att $L:: P_{\text {ara }}-m q s R A$ & This study \\
\hline ML3264 & $P_{l a c}-c f p$ & This study \\
\hline ML3265 & $\Delta 10 \mathrm{TA} P_{l a c}-c f p$ & This study \\
\hline ML3266 & $P_{l a c}-y f p$ & This study \\
\hline ML3267 & $\Delta 10 \mathrm{TA} P_{l a c}-y f p$ & This study \\
\hline
\end{tabular}

